\title{
THREE-DIMENS IONAL PLASMA SIMULATION MODELS AND THEIR APPLICATION TO MAGNETICALLY CONFINED PLASMAS
}

\section{PLASMA PHYSICS LABORATORY}

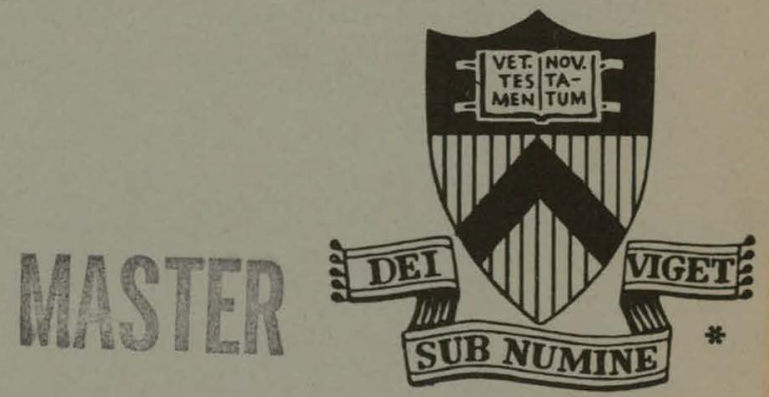

\section{PRINCETON UNIVERSITY PRINCETON, NEW JERSEY}

This work was supported by U. S. Fnergy Research and Development Administration Contract $\mathrm{E}(11-1)-3073$. Reproduction, translation, publication, use and disposal, in whole or in part, by or for the United States Government is permitted. 


\section{DISCLAIMER}

This report was prepared as an account of work sponsored by an agency of the United States Government. Neither the United States Government nor any agency Thereof, nor any of their employees, makes any warranty, express or implied, or assumes any legal liability or responsibility for the accuracy, completeness, or usefulness of any information, apparatus, product, or process disclosed, or represents that its use would not infringe privately owned rights. Reference herein to any specific commercial product, process, or service by trade name, trademark, manufacturer, or otherwise does not necessarily constitute or imply its endorsement, recommendation, or favoring by the United States Government or any agency thereof. The views and opinions of authors expressed herein do not necessarily state or reflect those of the United States Government or any agency thereof. 


\section{DISCLAIMER}

Portions of this document may be illegible in electronic image products. Images are produced from the best available original document. 
NOTICE

This report was prepared as an account of work sponsored by the United States Government. Neither the United States nor the United States Energy Research and Development Administration, nor any of their employees, nor any of their contractors, subcontractors, or their employees, makes any warranty, express

or implied, or assumes any legal liability or responsibility for the accuracy, completeness or usefulness of any information, apparatus, product or process disclosed, or represents that its use would not infringe privately owned rights.

Printed in the United States of America.

Available from

National Technical Information Service

U. S. Department of Commerce 5285 Port Royal Road

Springfield, Virginia 22151

Price: Printed Copy $\$ \ldots$; Microfiche $\$ 3.00$

NTIS

*Pages
$1-50$
$51-150$
$151-325$
$326-500$
$501-1000$

Selling Price

$\$ 4.00$

5.45

7.60

10.60

13.60 


\section{PRINCETON UNIVERSITY \\ Plasma Physics Laboratory}

\section{Three-Dimensional Plasma Simulation Models and}

Their Application to Magnetically Confined Plasmas

HIDEO OKUDA

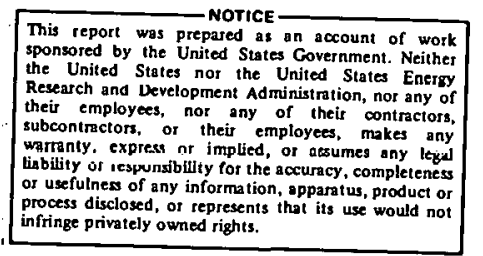

Lectures given at the College in Theoretical and Computational Plasma Physics, March 22 - April 9, 1977 in Trieste.

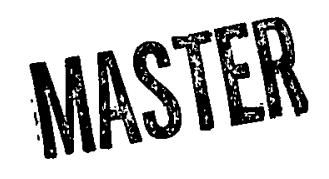


Three-dimensional plasma simulation models using particles have been described and applied to the various microscopic processes in a plasma in a magnetic field. The model makes use of the hybrid approach using eigenfunction expansion in one direction and multipole expansion on a twodimensional spatial grid introduced in the cross section of a plasma. The models for cylindrical and toroidal systems correctly reproduce the expected fluctuation spectrum in thermal equilibrium. Application to the study of anomalous plasma diffusion due to collisionless drift instabilities in a cylindrical plasma is shown. Some considerations are given as to how to construct a quasi-neutral particle simulation model and particle-fluid hybrid plasma model which eliminate the high frequency oscillations associated with the electrons.

\footnotetext{
* Lectures given at the college in Theoretical and Computational Plasma Physics, International Center for Theoretical Physics, March 22 - April 9, 1977, Trieste, Italy.
} 


\section{INTRODUCTION}

Since the pioneering works on one-dimensional plasma models by Buneman ${ }^{1}$ and Dawson ${ }^{2}$, plasma simulations using particle models have been developing rapidly in the area of both plasma physics and fusion applications. Due to the inherent nonlinearities associated with plasma dynamics, analytical approaches are rather limited and simulation methods can often provide clues to the extremely complicated nonlinear processes in the presence of strong plasma turbulence. Such examples are plasma heating due to parametric instabilities, anomalous diffusion associated with the convective cells and low-frequency microinstabilities in a magnetic field.

Particle simulations have also proved important in the area of fusion applications. Making use of the Monte Carlo method for the Fokker-Planck collisions, neoclassical diffusion has been proved for a toroidal plasma. study of neutral injection has been done using a simulation model taking into account the plasma inhomogeneities. In these calculations, the plasma induced self-consistent electric and magnetic fields are usually neglected.

Most of the self-consistent plasma simulations have been carried out in reduced dimensions with idealized boundary conditions. While some approximations and idealizations are inevitable for computer modeling complex plasma behavior in laboratory experiments, essential features of nonlinear 
plasma processes are often thiree-dimensional (six-dimensional phase space) in nature. This is especially true for a plasma in a magnetic field where the particle motions along and across the magnetic field often couple each other through nonlinear interactions.

It is the purpose of this lecture to introduce threedimensional plasma models for cylindrical and toroidal systems. ${ }^{3}$ While the models we consider are idealized compared with real experimental devices, it will be shown that full threedimensional simulations may be carried out using the presently available computers for the problems considered. After describing the mathematical properties of the model, the model has been applied to various plasma problems, in particular, heating and confinement of low-density plasmas in a strong magnetic field. They are lower hybrid wave heating, anomalous plasma diffusion due to collisionless drift instabilities in a cylindrical plasma and trapped particle instabilities in a toroidal system. These three-dimensional simulations are in essence equivalent to real laboratory experiments performed in computers and a great deal of information is available, some of which is inaccessible through laboratory experiments. We will then discuss a quasi-neutral particle simulation model and hybrid plasma model in which high-frequency oscillations associated with the electron inertia are eliminated while keeping the low-frequency ion fluctuations unmodified. 
The model is therefore particularly useful for studying the

- low frequency oscillations associated with ions such as ion sound waves.

The summary of this lecture is given in Sec. VIII.

\section{CYLINDRICAL PLASMA MODEL}

Let us consider a cylindrical plasma model of rectangular cross section immersed in a strong magnetic field as shown in Fig. 1. The model may be considered to correspond to linear laboratory devices such as Q-machines or even toroidal devices wherever applicable. When trying to model laboratory plasma devices, we note immediately that the presently available computers are much too small in capacity and too slow in speed. For example, we may use a 64 × 64 spatial grid in the cross section of a plasma column for solving the field equations and for pushing particles. This is a typical size for plasma simulations in two dimensions. For three-dimensional plasma models, the third direction along the main magnetic field must be included. Typically the length of the laboratory devices for confinement is much longer than the cross sectional dimension, typically more than ten times, so that the number of spatial grid in the axial direction would be much greater than 64 if the same resolution is required along the field lines. If this is the case, no available computers can handle full three-dimensional simulations due to their limited capacity. 
Fortunately, however, the resolution along the magnetic field may be much coarser than that across the field when modeling a confined plasma in a strong magnetic field. This is because the important physics for plasma confinement is often associated with the collective oscillations having very long wavelengths along the field Iines comparable to the machine lenqth, and short wavelenqths across them. Such examples are lower hybrid waves, drift and trapped particle instabilities and magnetohydrodynamic instabilities. When modeling these phenomena, one can neglect short wavelength fluctuations along the field lines and keep only the long wavelength modes. This is done most conveniently by expanding the field quantities in terms of Fourier harmonics along the field lines and across them, standard two-dimensional spatial grid is used. As will be shown later, keeping only 10 to 20 modes in the axial direction gives satisfactory results for the problems considered. Then the number of effective grid points required for the simulation is $64 \times 64 \times(10 \sim 20)$, which is much smallex than $64 \times 64 \times 640$, and may be stored in the fast core of a computer. The reason why we use Fourier harmonics instead of spatial grid in the axial direction is that too coarse grid can introduce numerical instabilities due to aliases. 4

Let us now outline the mathematical derivations of the model. We consider the electrostatic interactions only in 
this lecture, although extension to include more complex interactions is straightforward. Assume a particle has a finite size given by the gaussian charge density

$$
\begin{aligned}
\rho_{j}(x, y, z)=\frac{q_{j}}{(2 \pi)^{3 / 2} a_{x} a_{y} a_{z}} \exp [ & -\frac{\left(x-x_{j}\right)^{2}}{2 a_{x}^{2}}+\frac{\left(y-y_{j}\right)^{2}}{2 a_{y}^{2}} \\
& \left.+\frac{\left(z-z_{j}\right)^{2}}{2 a_{z}^{2}}\right]
\end{aligned}
$$

where $\left(x_{j}, y_{j}, z_{j}\right)$ is the location of the center of the $j-t h$ particle, $q_{j}$ is its total charge and $a_{x^{\prime}} a_{y}$ and $a_{z}$ are the particle size in three directions.

The Poisson equation will be

$$
\nabla^{2} \Phi=-4 \pi \sum_{j} \rho_{j}(x, y, z)
$$

where the summation over $j$ is over all the particles.

As described in the previous section, let us expand $\Phi(x, y, z)$ and $\rho(x, y, z)$ in terms of eigenfunctions $\exp (i k z)$ where $k_{z}=2 \pi n / L_{z} \cdot L_{z}$ is the length of the system which is assumed periodic in $\mathbf{z}$.

$$
\begin{aligned}
& \Phi(x, y, z)=\sum_{n=-N}^{N} \psi_{n}(x, y) \exp \left(i 2 \pi n z / L_{z}\right) \\
& \rho(x, y, z)=\sum_{n=-N}^{N} \rho_{n}(x, y) \exp \left(i 2 \pi n z / L_{z}\right)
\end{aligned}
$$

and $\mathrm{N}$ is the number of the modes in $\mathrm{z}$ kept in the model. 
$\rho_{n}(x, y)$ is found from Eq. (1) and Eq. (4).

$$
\begin{aligned}
\rho_{n}(x, y) & =\frac{1}{L_{z}} \int_{0}^{L} \rho(x, y, z) \exp \left(-i 2 \pi n z / L_{z}\right) d z \\
& =\frac{1}{2 \pi a a_{x} a_{y} L_{z}} \exp \left(-k_{z}^{2} a_{z}^{2} / 2\right) \sum_{j} q_{j}\left[-\frac{\left(x-x_{j}\right)^{2}}{2 a_{x}^{2}}+\frac{\left(y-y_{j}\right)^{2}}{2 a_{y}^{2}}\right] \\
& \cdot \operatorname{axp}\left(-i z_{z} z_{j}\right)
\end{aligned}
$$

Then the equation for $\phi_{\mathrm{n}}(\mathrm{x}, \mathrm{y})$ is found from Eq. (2), Eq. (3) and $\mathrm{Eq} \cdot(5)$.

$$
\left[\nabla_{x y}^{2}-\left(2 \pi n / L_{z}\right)^{2}\right] \phi_{n}(x, y)=-4 \pi \rho_{n}(x, y)
$$

Equation (6) is basically the Poisson equation in two dimensions and may be solved by means of fast Fourier transform using a two-dimensional spatial grid. To do so, let us again Fourier transform Eq. (6) with respect to $x$ and $y$. Assuming periodic boundary conditions for simplicity,

$$
-\left[\left(k_{x}^{2}+k_{y}^{2}\right)+\left(2 \pi n / L_{z}\right)^{2}\right] \bar{\phi}_{n}\left(k_{x}, k_{y}\right)=-4 \pi \bar{\rho}_{n}\left(k_{x}, k_{y}\right)
$$

where 


$$
\begin{aligned}
\bar{\rho}_{n}\left(k_{x}, k_{y}\right)= & \frac{1}{L_{x} L_{y}} \int_{0}^{L} \int_{0}^{L} \rho_{n}(x, y) \exp \left[-i\left(k_{x} x+k y\right)\right] d x d y \\
= & \frac{1}{L_{x}^{L} L_{z}^{L}} \exp \left[-\left(k_{x}^{2} a_{x}^{2}+k_{y}^{2} a_{y}^{2}+k_{z}^{2} a_{z}^{2}\right) / 2\right] \sum_{j} q_{j} \\
& \exp \left[-i\left(k_{z} z_{j}+k_{x} x_{j}+k_{y} y_{j}\right)\right] .
\end{aligned}
$$

Introducing a two-dimensional spatial grid in the $(x, y)$ plane, $\left(x_{j}, y_{j}\right)$ is expanded around its nearest grid point. writing $x_{j}=n_{j}^{x_{A}}+\delta x_{j}$ and $y_{j}=n_{j}^{y}+\delta y_{j}$, where $\left(n_{j}^{x}, n_{j}^{Y}\right)$ is the nearest grid point of the $j-t h$ particle, $\left(\delta x_{j}, \delta y_{j}\right)$ is the displacement and $\Delta$ is the grid space, exp $\left(-i k_{x} x_{j}\right)=\exp$ $\left(-i k_{x} n_{j}^{x} \Delta\right)\left(1-i k_{x} \delta x_{j}\right)$ and $\exp \left(-i k_{y} y_{j}\right)=\exp \left(-i k_{y} n_{j}^{Y} \Delta\right) \quad(1-$ ik $y^{\delta} y_{j}$ ) keeping up to the dipole moments for each particle. Then $\vec{\rho}_{n}\left(k_{x}, k_{y}\right)$ will be

$$
\begin{aligned}
\bar{\rho}_{n}\left(k_{x^{\prime}} k_{y}\right) & =\frac{1}{L_{x^{L} y_{z} L_{z}}} \exp \left[-\left(k_{x}^{2} a_{x}^{2}+k_{y}^{2} a_{y}^{2}+k_{z}^{2} a_{z}^{2}\right) / 2\right] \sum_{s=1}^{L_{x}} \sum_{s^{\prime}=1}^{L_{y}} \\
& \left(\rho_{s s^{\prime}}-i k_{x^{\prime}} \delta \rho_{s^{\prime} s^{\prime}}^{x^{\prime}}-i k_{y} \delta \rho_{s s^{\prime}}^{\left.y^{\prime}\right)} \exp \left[-i 2 \pi\left(\frac{s \ell}{r_{x}}+\frac{s^{\prime} m}{L_{y}}\right)\right]\right.
\end{aligned}
$$

where

and

$$
\begin{aligned}
& \rho_{s s^{\prime}}=\sum_{j \varepsilon\left(s, s^{\prime}\right)} \bar{q}_{j} \exp \left(-i k_{z_{j}} z_{j}\right) \\
& \delta \rho_{s^{\prime}}^{x}=\sum_{j \in\left(s^{\prime} s^{\prime}\right)} q_{j} \exp \left(-i k_{z^{\prime}} z_{j}\right)\left(x_{j}-s \Delta\right)
\end{aligned}
$$

$$
\delta \rho_{s s^{\prime}}^{Y}=\sum_{j \varepsilon\left(s, s^{\prime}\right)} q_{j} \exp \left(-i k_{z^{2} j^{\prime}}\right)\left(y_{j}-s^{\prime} \Delta\right)
$$


$L_{x}$ and $L_{y}$ are the length of the system in $x$ and $y$ in terms of the grid size $\Delta$ and $k_{x}=2 \pi l / L_{x}$ and $k_{y}=2 \pi m / L_{y}$ for a periodic system. We note Eq. (8) is the fast Fourier transforms of monopole $\rho_{s s^{\prime}}$ and dipole charge densities $\delta \rho_{s s^{\prime}}^{\mathrm{x}} \delta \rho_{\text {ss }}^{\mathrm{Y}}$, on the grid points. It is straightforward to find the potential $\bar{\Phi}_{n}\left(k_{x}, k_{y}\right)$ from Eq. (7) and its inverse transform

$$
\bar{\phi}_{n}\left(s \Delta, s^{\prime} \Delta\right)=\sum_{m=1}^{L_{x}} \sum_{\ell=1}^{L_{y}} \phi_{n}\left(k_{x}, k_{y}\right) \exp \left[i 2 \pi\left(\frac{s m}{L_{x}}+\frac{s^{\prime} \ell}{L_{y}}\right)\right] \text {. }
$$

Finally, the total potential will be given by summing over $\mathrm{n}$ as given by Eq. (3).

Let us now calculate the force on a finite-size particle which is used in the equation of motion. The electric field force on the $j-t h$ particle will be given by

$$
\begin{aligned}
\underset{\vdots}{F}\left(x_{j}, y_{j}, z_{j}\right)= & q_{j} \int \underset{z}{E(x, y, z)} \frac{1}{(2 \pi)^{3 / 2} a_{x} a_{y} a_{z}} \\
& \exp \left[-\left(\frac{\left(x-x_{j}\right)^{2}}{2 a_{x}^{2}}+\frac{\left(y-y_{j}\right)^{2}}{2 a_{y}^{2}}\right.\right. \\
& \left.\left.+\frac{\left(z-z_{j}\right)^{2}}{2 a_{z}^{2}}\right)\right] d x d y d z
\end{aligned}
$$

where $\underset{\sim}{\mathrm{E}}$ is calculated from $\Phi$ and is assumed to take the form of

$$
\underset{\sim}{E}(x, y, z)=\sum_{n=-N}^{N} \underset{\sim n}{E}(x, y) \text { exp }\left(-2 \pi i n z / L_{z}\right)
$$


The $z$-integration can be carried out to find the force in the $x$-direction

$$
\begin{aligned}
F_{x}\left(x_{j}, y_{j}, z_{j}\right)= & \frac{q_{j}}{2 \pi a_{x} a_{y}} \sum_{n=-N}^{N} \exp \left(-k_{z}^{2} a_{z}^{2} / 2\right) \exp \left(i k_{z} z_{j}\right) \iint E_{n x}(x, y) \\
& \exp \left[-\left(\frac{\left(x-x_{j}\right)^{2}}{2 a_{x}^{2}}+\frac{\left(y-y_{j}\right)^{2}}{2 a_{y}^{2}}\right)\right] d x d y .
\end{aligned}
$$

Since $\mathrm{E}_{\mathrm{nx}}(\mathrm{x}, \mathrm{y})$ is defined on the two-dimensional grid points, $F_{x}\left(x_{j}, y_{j}, z_{j}\right)$ is Fourier transformed with respect to $\left(x_{j}, y_{j}\right)$.

$$
\begin{aligned}
F_{x}\left(k_{x}, k_{y}, z_{j}\right)= & q_{j} \sum_{n=-N}^{N} \exp \left(-k_{z}^{2} a_{z}^{2} / 2\right) \exp \left(i k_{z} \dot{z}_{j}\right) E_{n x}\left(k_{x}, k_{y}\right) \\
& \exp \left[-\left(k_{x}^{2} a_{x}^{2} / 2+k_{y}^{2} a_{y}^{2} / 2\right)\right]
\end{aligned}
$$

where $E_{n x}\left(k_{x}, k_{y}\right)$ is found from $\phi_{n}\left(k_{x}, k_{y}\right) \operatorname{directly} \cdot F_{x}\left(s \Delta, s^{\prime} \Delta, z_{j}\right)$ is found from the inverse fast Fourier transform and $F_{x}\left(x_{j}, y_{j}, z_{j}\right)$ is found from

$$
F_{x}\left(x_{j}, y_{j}, z_{j}\right)=F_{x}\left(n_{j} x_{,} n_{j}^{y_{j}} z_{j}\right)+\delta x_{j} \frac{\partial F_{x}}{\partial x}+\delta y_{j} \frac{\partial F_{y}}{\partial y}
$$

keeping up to the dipole terms. The derivatives of $F_{\mathbf{x}}$ are evaluated at the nearest grid point and are calculated by finite difference scheme.

For the simulations of drift and trapped particle instabilities and lower hybrid heating, ions are pushed using the full Lorentz force while the electrons are assumed to 
be guiding center particles. A predictor-corrector two-step method is used. In the finite difference scheme, equations are written for the ions

$$
\begin{aligned}
\frac{{\underset{\sim}{j}}_{j}^{t+\Delta t / 2}-{\underset{\sim}{v}}_{j}^{t-\Delta t / 2}}{\Delta t} & =\frac{q_{j}}{m_{i}}\left[\underset{\sim}{E}\left({\underset{\sim}{x}}_{j}^{t}\right)+\frac{{\underset{\sim}{j}}_{j}^{t+\Delta t / 2}+{\underset{\sim}{j}}_{j}^{t-\Delta t / 2}}{2 c} \times \underset{\sim}{B}\left(x_{j}^{t}\right)\right] \\
\frac{{\underset{x}{j}}_{j}^{t+\Delta t}-\bar{x}_{j}^{t}}{\Delta t} & =\underset{j}{t+\Delta t / 2}
\end{aligned}
$$

and for the electrons

$$
\begin{aligned}
& \frac{v_{j \|}^{*}: v_{j}^{t+\Delta t}-v_{j}^{t-\Delta t}}{2 \Delta t}=-\frac{e}{m_{e}} E_{\|}(\underset{\sim j}{t}) \\
& \frac{x_{j}^{*+\Delta t}-x_{j}^{t-\Delta t}}{2 \Delta t}={\underset{\sim}{j}}_{j}^{t} \\
& \frac{v_{j \|}^{t+\Delta t}-v_{j \|}^{t}}{\Delta t}=-\frac{e}{m_{e}} \frac{E_{\|}\left(x_{j}^{t}\right)+E_{j}^{*} x_{j}^{* t+\Delta t}}{2} \\
& \frac{{\underset{\sim}{j}}_{j}^{t+\Delta t}-x_{j}^{t}}{\Delta t}=\frac{1}{2}\left(v_{\sim j}^{t}+{\underset{\sim}{v}}_{j}^{\star t+\Delta t}\right) .
\end{aligned}
$$

Note that the scheme described requires the solution of the Poisson equation twice per one time step.

\section{TEST OF THE CYLINDRICAL MODEL}

Let us briefly describe the test of the model and some of the basic properties associated with it. Consider first a slab system of $64 \times 32 \times 32$ cube. The plasma is assumed 
periodic and homogeneous in a uniform external magnetic field in z-direction. Initially $2^{12}$ ions and electrons are loaded uniformly in the cube. $\Omega_{e} / \omega_{p e}=1, m_{i} / m_{e}=25, T_{e} / T_{i}=1$, $\lambda_{e} / \Delta=2, a_{x}=a_{y}=a_{z}=1$ and $\omega_{p e} \Delta t=1$ were used where $\Omega_{e}$ $\omega_{\mathrm{pe}} \lambda_{e^{\prime}} \Delta$ and $\Delta t$ are electron gyrofrequency, electron plasma frequency, electron Debye length, grid space and the integration step. Both ions and electrons had Maxwell velocity distributions. Since the plasma is in thermal equilibrium after the relaxation of the initial loading, one can predict the spectral distribution of the electric field fluctuations using statistical mechanics in thermal equilibrium. It is given by

$$
\frac{\mathrm{E}_{\mathrm{k}}^{2}}{8 \pi}=\frac{\mathrm{T}}{2} \frac{1}{1+\mathrm{k}^{2} \lambda_{\mathrm{D}}^{2} \exp } \frac{}{\left(\mathrm{k}^{2} \mathrm{a}^{2}\right)}
$$

where $\mathrm{E}_{\mathrm{k}}^{2} / 8 \pi$ is the electric field energy associated with the mode $\underset{\sim}{\mathrm{k}}=\left(\mathrm{k}_{\mathrm{x}}, \mathrm{k}_{\mathrm{y}}, \mathrm{k}_{\mathrm{z}}\right)=\left(2 \pi \mathrm{m} / \mathrm{L}_{\mathrm{x}}, 2 \pi \mathrm{n} / \mathrm{L}_{\mathrm{y}}, 2 \pi \ell / \mathrm{L}_{\mathrm{z}}\right)$. Figure 2 indicates the time averaged measurement from $\omega_{\mathrm{pe}} t=0$ to $\omega_{\text {pe }} t=50$ for comparison to the theory. The measurement agrees quite well with the prediction shown by the solid curve. Nine modes ( $n=0, \pm 1, \pm 2, \pm 3, \pm 4)$ are kept in the calculation and the total energy conservation is about $0.5 \%$.

Several test runs are made to study the noise properties of plasmas of large volume comparable to small laboratory devices. A system of $64 \times 64 \times 680$ cube is chosen and a $64 \times 64$ two-dimensional grid together with nine modes $(\mathrm{n}=0$, $\pm 1, \pm 2, \pm 3, \pm 4$ ) is used. The plasma is uniform and triply 
periodic with a uniform magnetic in $z$-direction. Note that the size of the system is ten times longer than the cross section simulating laboratory devices. The parameters of the simulations are $\mathrm{m}_{\mathrm{i}} / \mathrm{m}_{\mathrm{e}}=400, \mathrm{~T}_{\mathrm{e}} / \mathrm{T}_{\mathrm{i}}=4, \lambda_{\mathrm{e}} / \Delta=2$, $\Omega_{\mathrm{e}} / \omega_{\mathrm{pe}}=5, \omega_{\mathrm{pe}} \Delta t=4$ and $\mathrm{a}_{\mathrm{x}} / \Delta=\mathrm{a}_{\mathrm{y}} / \Delta=1$ and $\mathrm{a}_{\mathrm{z}} / \Delta=25$. Note that the finite size particles are elongated along the field lines. Only the modes whose wavelengths along the field lines are very long $\left(k_{1}>k_{\|}\right)$are retained in the model. This will serve to use a large time step of integration since the highest frequency of the system will be $\omega_{\text {pe }} k / / k$ or $\omega_{p i}$ which is much smaller than $\omega_{\text {pe }}$ itself. $^{5}$ Electron gyrofrequency is also eliminated by using the guiding center drift approximations across the magnetic field.

Figure 3 shows the random fluctuation field energy for the casc of $2^{18}$ and $2^{19}$ total number of simulation particles. The noise level is $5 \times 10^{-4} \sim 10^{-3}$ of the kinetic energy and fluctuates around its mean value. The total energy conservation is found to be quite good, i.e., less than $0.1 \%$ and the system is quite stable in spite of the use of only nine modes in $z$. Note that the plasma volume of this example is comparable to that of small laboratory devices such as Qmachines and that one can simulate quite a few interesting physics using the present model. The model has been applied to plasma heating due to lower hybrid waves and anomalous diffusion associated with the collisionless drift instabilities. The latter is discussed in some detail in Sec.: VI. 
The model described here has been implemented on both IBM 360/91 at Princeton and CDC 7600 at Livermore. Typically, the code requires $150 \sim 200 \mathrm{~K}$ words memory including all the diagnostics with the particles are stored on the disks. It takes $50 \sim 100 \mu$ seconds per particle per step on the CDC 7600 which requires several hours for a typical production run.

\section{TOROIDAL PLASMA MODEL}

Let us briefly describe the simulation model for a toroidal system where essentially the same technique can be used. We first discuss how to solve the Poisson equation in a toroidal system shown in Fig. 4. Using a cylindrical coordinate, the Poisson equation takes the form of

$$
\frac{\partial^{2} \phi}{\partial r^{2}}+\frac{1}{r} \frac{\partial \Phi}{\partial r}+\frac{1}{r^{2}} \frac{\partial^{2} \phi}{\partial \phi^{2}}+\frac{\partial^{2} \phi}{\partial z^{2}}=-4 \pi \rho(r, \phi, z)
$$

where $\Phi(r, \phi, z)$ is the electrostatic potential.

Now expanding $\Phi$ and $\rho$ in the toroidal direction,

$$
\begin{aligned}
& \phi(r, \phi, z)=\sum_{n=-N}^{N} p_{n}(r, z) \exp (\text { in } \phi) \\
& \rho(r, \phi, z)=\sum_{n=-N}^{N} n(r, z) \exp (\text { in } \psi)
\end{aligned}
$$

Eq. (10) is reduced to

$$
\frac{\partial^{2} \Phi_{n}}{\partial r^{2}}+\frac{1}{r} \frac{\partial \Phi_{n}}{\partial r}-\frac{n^{2}}{r^{2}} \Phi_{n}+\frac{\partial^{2} \Phi_{n}}{\partial z^{2}}=-4 \pi \rho_{n} \text {. }
$$


To see how the multipole expansion method works for the toroidal system, let us calculate $\Phi_{n}(r, z)$ first. Assuming a particle has a gaussian charge density in the cylindrical coordinate,

$\rho_{j}(r, \phi, z)=\frac{q_{j}}{(2 \pi)^{3 / 2} a_{z} a_{r^{r} j_{\phi}}} \exp \left[-\left(\frac{\left(r-r_{j}\right)^{2}}{2 a_{r}^{2}}+\frac{\left(\phi-\phi_{j}\right)^{2}}{2 a_{\phi}^{2}}+\frac{(z-z)^{2}}{2 a_{z}^{2}}\right)\right]$

$n_{n}(r, z)=\frac{l}{2 \pi} \int_{0}^{2 \pi} \rho(r, \phi, z) e^{-i n \phi} d \phi$

$$
=\frac{\exp \left(-n^{2} a_{\phi}^{2} / 2\right)}{(2 \pi)^{2} a_{r} a_{z}} \sum_{j} \frac{q_{j}}{r_{j}} \exp \left[-\left(\frac{\left(r-r_{j}\right)^{2}}{2 a_{r}^{2}}+\frac{\left(z-z_{j}\right)^{2}}{2 a_{z}^{2}}+i n \phi_{j}\right)\right]
$$

where $a_{r}, a_{\phi}$ and $a_{z}$ are the particle size in the $(r, \phi, z)$ coordinate.

Fourier transforming $\rho_{n}(r, z)$ with respect to $z$, we find

$$
\begin{aligned}
\rho_{n}\left(r, k_{z}\right) & =\frac{1}{L_{z}} \int_{0}^{I_{1} \cdot z} \rho_{n}(r, z) \exp \left(-i k_{z} z\right) d z \\
& =\frac{\exp \left(-k_{z}^{2} a_{z}^{2} / 2-n^{2} a_{\phi}^{2} / 2\right)}{(2 i)^{3 / 2} a_{r} L_{z}} \sum_{j} \frac{q_{j}}{r_{j}}\left[-i\left(n \phi_{j}+k_{z} z\right)\right] \exp \left[-\left(r-r_{j}\right)^{2} / 2 a_{r}^{2}\right] \\
& =\frac{\exp \left(-k_{z}^{2} a_{z}^{2} / 2-n^{2} a_{\phi}^{2} / 2\right)}{(2 \pi)^{3 / 2} a_{r} L_{z}} \sum_{j} q_{j} \exp \left[-i\left(n \phi_{j}+k_{z} n_{j}^{2} \Delta z\right)\right]\left(1-i k_{z} \delta z_{j}\right)^{\prime} \\
& \cdot \exp \left[-\left(r-r_{j}\right)^{2} / 2 a_{r}^{2}\right] \frac{1}{r_{j}} .
\end{aligned}
$$


$-15-$

While the trigonometric functions are not the eigenfuncLions in the radial direction, let us calculate $\rho_{n}\left(k_{r}, k_{z}\right)$ to: determine the charge density on the radial grid in terms of the multipole expansion method.

$$
\begin{aligned}
& \bar{\rho}_{n}\left(k_{r}, k_{z}\right)=\frac{1}{L_{R}} \int_{0}^{L_{R}} \rho_{n}\left(R_{1}+s, k_{z}\right) \exp \left(-i k_{r} s\right) d s \\
& =\frac{\exp \left(-k_{z}^{2} a^{2} / 2-n^{2} a_{\phi}^{2} / 2-k_{r}^{2} a_{r}^{2} / 2\right)}{2 \pi L_{R}{ }^{L}{ }^{2} j} \sum_{j} q_{j} \exp \left[-i \quad\left(n \phi_{j}+\right.\right. \\
& \left.\left.k_{z} n_{j}^{z} \Delta_{z}\right)\right] \quad\left(1-i k_{z}{ }^{i z_{j}}\right) \exp \left[-i k_{r}\left(r_{j}-R_{l}\right)\right]
\end{aligned}
$$

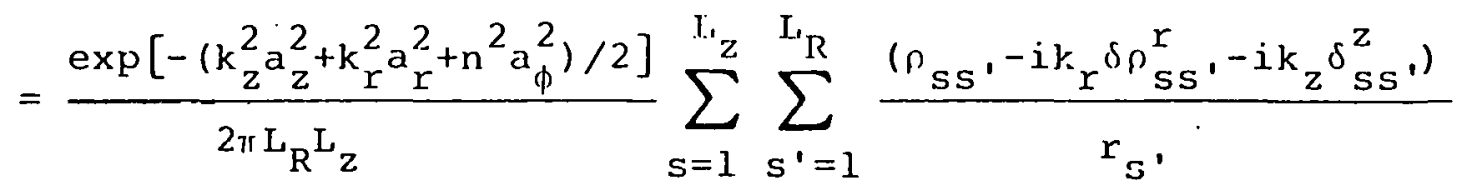

$$
\begin{aligned}
& \exp \left(-i 2 \pi s \ell / L_{z}\right) \exp \left(-i 2 \pi s^{\prime} \ell^{\prime} / L_{R}\right)
\end{aligned}
$$

$$
\begin{aligned}
\rho_{s s^{\prime}} & =\sum_{j \in\left(s, s^{\prime}\right)} q_{j} \exp \left(-i n \phi_{j}\right) \\
\delta \rho_{s^{\prime}}^{z} & =\sum_{j \in\left(s, s^{\prime}\right)} q_{j} \exp \left(-i n \phi_{j}\right) \quad\left(z_{j}-s \Delta z\right)
\end{aligned}
$$

and

$$
\delta \rho_{s s^{\prime}}^{r}=\sum_{j \varepsilon\left(s, s^{\prime}\right)} q_{j} \exp \left(-i n \phi_{j}\right)\left(r_{j}-R_{\perp}-s^{\prime} \Delta r\right)
$$


Inverting the finite Fourier transform of $\rho_{n}\left(k_{r}, k_{z}\right)$ with respect to $k$, the Poisson equation finally becomes

$$
\left[\frac{d^{2}}{d r^{2}}+\frac{1}{r} \frac{d}{d r}-\left(\frac{n^{2}}{r^{2}}+k_{r}^{2}\right)\right] \phi_{n}\left(r, k_{z}\right)=-4 \pi \rho_{n}\left(r, k_{z}\right)
$$

This equation (11) has been solved by using the cubic spline technique which is accurate up to $(\Delta r)^{3}$.

Let us calculate the force on a finite-size particle.

The clectric field force on a particle at $\left(r_{j}, \phi_{j}, z_{j}\right)$ is given

by

$$
\begin{aligned}
\underset{\sim}{F}\left(r_{j}, \phi_{j}, z_{j}\right)= & q_{j} \int \underset{\sim}{E}(r, \phi, z)(2 \pi)^{-3 / 2}\left(a_{z} a_{r} r_{j} a_{\phi}\right)^{-1} \\
& \exp \left[-\left(r-r_{j}\right)^{2} / 2 a_{r}^{2}-\left(z-z_{j}\right)^{2} / 2 a_{z}^{2}-\left(\phi-\psi_{j}\right)^{2} / 2 a_{\phi}^{2}\right] \text { rdrdwdz }
\end{aligned}
$$

where $\underset{\sim}{\mathrm{E}}$ is calculated from $\Phi$ and is given by, for example,

$$
E_{z}(r, \phi, z)=\sum_{n=-N}^{N} E_{n}(r, z) \exp (\text { in } \phi)
$$

then

$$
\begin{aligned}
F_{z}\left(r_{j}, \phi_{j}, z_{j}\right) & =\sum_{n=-N}^{N} q_{j} \exp \left(-n^{2} a_{\phi}^{2} / 2\right) \exp \left(i n \phi_{j}\right)\left(2 \pi a_{r} a_{z}\right)^{-1} \int E_{n z}(r, z) \\
& \cdot \exp \left[-\left(z-z_{j}\right)^{2} / 2 a_{z}^{2}-\left(r-r_{j}\right)^{2} / 2 a_{r}^{2}\right] \operatorname{drdz} .
\end{aligned}
$$

$F_{z}\left(r_{j}, \phi_{j}, z_{j}\right)$ is Fourier transformed with respect to $\left(r_{j}, z_{j}\right)$ 


$$
\begin{aligned}
F_{z}\left(k_{x}, \phi_{j}, k_{z}\right)= & \sum_{n=-N}^{N} q_{j} \exp \left[-\left(k_{z}^{2} a_{z}^{2}+k_{r}^{2} a_{r}^{2}+n^{2} a_{\phi}^{2}\right) / 2\right] q_{j} \exp \left(i n \phi_{j}\right) \cdot \\
& E_{n z}\left(k_{r}, k_{z}\right)
\end{aligned}
$$

The force on the $j$-th particle is calculated from the inverse transform of Eq. (12) and then expanded around the nearest grid point. Writing $r_{j}=n_{j} \Delta r+\delta r_{j}$ and $z_{j}=n_{j} \Delta z+\delta z_{j}$, $F_{z}\left(r_{j}, \phi_{j}, z_{j}\right)=F_{z}\left(n_{j}^{r} \wedge r, \phi_{j} \cdot n_{j}^{z} \Delta z\right)+\delta r_{j} \frac{\partial F}{\partial r}+\delta z_{j} \frac{\partial F}{\partial z}$.

The evolution of the system is obtained by pushing ions with the Lorentz force (9) and integrating the guiding center drift equations for electrons. The electron guiding center equations are given by

$$
\begin{aligned}
& \frac{\dot{\mathrm{u}} \underset{\sim}{\mathrm{r}}}{\mathrm{dt}}=\frac{(\underset{\sim}{\mathrm{v}} \cdot \underset{\sim}{\mathrm{B}})}{\mathrm{B}^{2}} \underset{\sim}{\mathrm{B}}+\frac{\underset{\sim}{\mathrm{E}} \times \underset{\sim}{\mathrm{B}}}{\mathrm{B}^{2}}-\frac{1}{\Omega_{\mathrm{e}^{B}}}\left(\frac{\mu \mathrm{B}}{\mathrm{m}_{\mathrm{e}}}+v_{\|}^{2}\right) \underset{\sim}{\mathrm{B}} \times v_{B} \\
& \frac{d v_{U}}{d t}=-\frac{q}{m_{e}} \frac{\underset{\sim}{E} \cdot \underline{B}}{B}-\frac{1}{m_{e} B} \underset{\sim}{B} \cdot V(\mu B) \equiv F \| \\
& \mu=\frac{m v_{\perp}^{2}}{2 B}=\text { constant }
\end{aligned}
$$

where

$$
\mathrm{v}_{\|}=\frac{\stackrel{v}{\mathrm{v}} \underline{\underline{B}}}{\mathrm{~B}}
$$


The integration scheme used is a two-step predictorcorrector scheme because the standard leap-frog scheme is not applicable. Symbolically,

$$
\begin{aligned}
& \vec{\sim}_{n+1}={\underset{\sim}{r}}_{n-1}+2 \Delta t \underset{\sim}{v}\left({\underset{\sim}{r}}_{n}, t_{n}\right) \\
& \bar{v}_{\| n+1}=v_{\| n-1}+2 \Delta t F_{\|}\left(\dot{s}_{n}, \dot{t}_{n}\right) \\
& \stackrel{r}{\sim}_{n+1}=\stackrel{r}{\sim}_{n}+\left(\underset{v}{v}\left(r_{n}, t_{n}\right)+\underset{v}{v}\left(\overline{\dot{r}}_{n+1}, t_{n+1}\right)\right) \Delta t / 2 \\
& \left.v_{\| n+1}=v_{\| n}+\left(F_{\|}{\left(\dot{r}_{n}, t_{n}\right)+F}_{\|} \stackrel{\sim}{r}_{n+1}, t_{n+1}\right)\right) \Delta t / 2
\end{aligned}
$$

Note that this scheme requires two solutions of the Poisson equation per one time step.

\section{TEST OF THE TOROIDAL MODEL}

In the toroidal model the imposed magnetic field is taken to be $\underset{\sim}{\mathrm{B}}=\hat{e}_{\theta} B_{\theta}+\hat{e}_{\phi^{B}} \phi^{\prime}$ where

$$
\begin{aligned}
& B_{\phi}(r)=B_{\phi}^{(0)} R_{0} / r \\
& B_{\theta}=\bar{r}_{\phi}^{(0)} / q(\bar{r}) R_{O}=\frac{\bar{r} B_{\phi}^{(0)}}{q{ }^{(0)} R_{O}}\left(1-\frac{\bar{r}^{-2}}{2 \bar{a}^{-2}}\right)
\end{aligned}
$$

and

$$
r=R_{0}+\bar{r} \cos \theta
$$


The coordinate $(\bar{r}, \theta)$ measures the position in the cross sectional plane of the torus with $\bar{r}$ the distance from the minor axis and $\theta$ measured from the toroidal plane to the inside. $R_{0}$ and $\bar{a}$ are the major and minor radius respectively, and $\mathrm{q}^{(0)}$ is the safety factor and $\mathrm{B}_{\phi}^{(0)}$ the toroidal field at the minor radius. The plasma is assumed to be bounded due to the conducting walls which reflect particles. Initially the plasma density and temperature profiles are taken to be gaussians with, e.g.,

$$
n(\bar{r})=\frac{2 e^{-\bar{r}^{2} / C^{-} 2}}{c^{-2}\left(1-e^{-1 / C}\right)}
$$

The constant $\mathrm{C}$ characterizes the degree of nonuniformity. The plasma is locally Maxwellian. Figure 5 shows the fluctuation spectrum averaged over the r-direction for different modes of

$$
\left(\mathrm{k}_{\mathrm{z}}, \mathrm{k}_{\phi}\right)=\left(\frac{2 \pi \ell}{\mathrm{L}_{\mathrm{z}}}, \frac{\mathrm{n}}{\mathrm{R}_{\mathrm{O}}}\right)
$$

The size of the torus is taken $\bar{a}=16, R_{0}=40$. There are 9 modes $(\mathrm{n}=0, \pm 1, \pm 2, \pm 3, \pm 4)$ in the toroidal direction $\phi$ and $32 \times 32$ spatial mesh was employed in the $r-z$ cross sectional plane. The other parameters of the run are $2^{14}$ particles for each species, $\Omega_{\mathrm{e}} / \omega_{\mathrm{pe}}=4, \mathrm{~T}_{\mathrm{e}} / \mathrm{T}_{\mathrm{i}}=10, \mathrm{~m}_{\mathrm{i}} / \mathrm{m}_{\mathrm{e}}=100$, 
$\lambda_{\text {De }}=2$, a (isotropic particle size) $=1$ and $\omega_{\text {pe }} \Delta t=1$, $\mathrm{C}_{\mathrm{Ti}}=\mathrm{C}_{\mathrm{Te}}=0.25, \mathrm{C}_{\mathrm{ni}}=\mathrm{C}_{\mathrm{ne}}=0.5$, where $\Omega_{\mathrm{e}}$ is the electron gyrofrequency at the minor radius, $\omega_{p e}$ is the average electron plasma frequency, $C_{T}$ and $C_{n}$ denote temperature and density nonuniformity and $\mathrm{T}_{\mathrm{e}} / \mathrm{T}_{i}=10$ at the center of minor radius.

As we can see, the aqreement between the thenretiral predictions and the numerical results averaged over $\omega_{\text {pe }} t=$ $50 \sim 100$ is quite satisfactory for short-wavelength modes. The long-wavelength modes generated by the $\nabla \mathrm{B}$.drift of the ions have not yet reached the equilibrium, because the computations was too short for the electron flow along the field lines to compensate the ions. The theoretical fiuctuation spectrum averaged over the radial direction for a finitesize particle system is given by

$$
\int \mathrm{rds}: \frac{|E(r, n, \ell)|^{2}}{8 \pi} /\left(\frac{T}{2} L_{R}\right)=\frac{1}{2 \pi \lambda} \int_{0}^{\pi \lambda_{D}} \frac{d x}{1+\left(y^{2}+x^{2}\right) \exp \left[\left(y^{2}+x^{2}\right) a^{2} / \lambda_{D}^{2}\right]}
$$

where $y^{2}=\left(n^{2} / R_{0}^{2}+\ell^{2} \Delta k_{z}^{2}\right) \lambda_{D}^{2}$ and is shown by the solid line. 'l'he error for the total energy conservation at the end of the calculation $\left(\omega_{p e} t=100\right)$ is about $1 \%$. This may be due to too large time step of integration, too few particles per pebye sphere, the reflecting boundary walls which exert external force on the particles. Because the toroidal model is more complicated than the slab model, the former takes about $50 \%$ more computations than the latter. 


\section{APPLICATION OF THE MODEL}

After testing the three-dimensional models in cylindrical and toroidal geometries, the models have been used for the study of lower hybrid heating, anomalous plasma diffusion due to collisionless drift instabilities and trapped particle instabilities in toroidal geometry.

Here we report our recent results of numerical simulations on collisionless drift instabilities using large scale fully three-dimensional cylindrical plasma models developed earlier. Theoretical interpretations of the observed results on plasma turbulence and diffusion are also given. Driftwave turbulence has been of current interest in toroidal confinement devices such as tokamaks, ${ }^{6}$ stellarators, ${ }^{7}$ and internal ring devices 8,9 because of the strong correlation between the observed density fluctuations and anomalous plasma transport.

The simulation model used is a straight cylinder in a uniform external magnetic field $B_{0}$ along the $z$ direction with its length $\mathrm{L}_{\mathrm{L}} / \rho_{\mathrm{i}}=640, \rho_{\mathrm{i}}=\left(\mathrm{T}_{\mathrm{i}} / \mathrm{m}_{\mathrm{i}}\right)^{1 / 2 / \Omega_{i}}$ being the ion qyroradius. In the cross section, a $64 \times 64\left(L^{2}\right)$ spatial grid is used. for numerical computation with its physical length $L / \rho_{i}=32$. The plasma is periodic in $z$ while it is surrounded by a conducting wall at the boundary of the cross section. Initially both ions and electrons are Maxwellian velocity

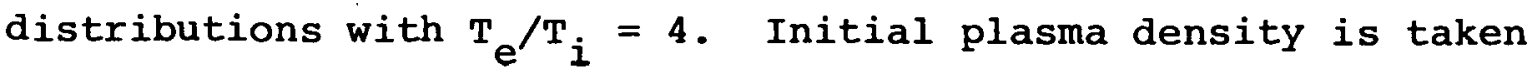
to be $n_{e}(r)=n_{i}(r)=n_{0} \exp \left(-4 r^{2} / a^{2}\right)$ with the average density given by $\Omega_{\mathrm{e}} / \omega_{\text {pe }}=5, \mathrm{~m}_{\mathrm{i}} / \mathrm{m}_{\mathrm{e}}=100, \mathrm{a}=\mathrm{L} / 2$ and there is 
no initial temperature gradient. Seven Fourier modes $\mathrm{n}=0$, $\pm 1, \pm 2$, and \pm 3 are kept in the $z$ direction with $k_{z}=2 \pi n / L_{z}$. Linear theory predicts that the collisionless drift instability (universal mode) is strongly unstable for $\mathrm{n}= \pm 1$ and becomes stable rapidly with increasing $|n|$ due to the onset of ionLandau damping. This situation is commonly observed in Qdevices. In terms of physical size, the simulation plasma is certainly smaller than a tokamak plasma but may be larger than a conventional Q-device and one can study quite a few problems with the model using realistic plasma parameters.

Let us first look at the gross behavior of the instability. Figure 6 shows the particle diffusion, heat transfer, electron velocity distribution, and radial mode structure associated with the instability. While ions and electrons diffuse more or less together in the early stage, we see charge separation built up at a later stage due to the difference of radial $\mathrm{CE}_{\theta} / \mathrm{B}$ drifts of ions and electrons where $\mathrm{E}_{\theta}$ is the azimuthal electric field associated with the drift wave. As is shown later, this charge separation generates strong two-dimensional electric fields which in turn excite large amplitude convective cells $\left(\omega=0, \mathrm{k}_{\|}=0\right)$. The observed anomalous particle diffusion is mainly due to drift instabilities at the early stage and at the later stage it is due to nonlinearly excited convective cells, which enhance diffusion even when the drift instability is quenched. 
The electron-parallel temperature $\mathrm{T}_{\mathrm{e}}$. rapidly decreases for $r / a>0.5$ due to inverse-Landau damping and increases for $r / a<0.5$ due to the absorption of wave energy associated with the radial convection. The electron-parallel velocity distribution steepens due to the inverse-Landau damping. 10 Ionperpendicular temperature $\mathrm{T}_{\mathbf{I}^{\perp}}$ increases due to the accelaration across the magnetic field associated with the shortwavelength turbulence due to the convective cells. Ion-parallel temperature $\mathrm{T}_{i} \|$ changes little because ion-Landau damping is weak. A typical drift-wave radial mode structure $(n=1$, $\mathrm{m}=3)\left|\mathrm{e} \phi(\mathrm{r}) / \mathrm{T}_{\mathrm{e}}\right|$ shows a peak near $\mathrm{r} / \mathrm{a}=0.5$ at the early stage, consistent with the initial density profile. After reaching the maximum amplitude of 10\% at $\omega_{\mathrm{pe}} t=1040$, it begins to level off due to nonlinear excitation of convective cells $(n=0)$.

Figure 7 shows more detailed diagnostics of the instability; spectral distribution $\left|\mathrm{E}^{2}(\mathrm{~m})\right|$ and $\left|\mathrm{E}^{2}(\mathrm{n})\right|$ and power spectrum $P_{m n}(\omega, r)$ with respect to $k_{\theta}$ and $k_{z}$ where $m$ is the azimuthal mode number with $k_{\theta}=m / r .\left|E^{2}(m)\right|$ indicates typical spectral profile for drift turbulence as seen in the experiments; ${ }^{6}$ it peaks around $k_{\theta} \rho_{i}=0.3 \sim 0.6$ corresponding to $\mathrm{m}=2,3$, and 4 modes for this case. The amplitude decreases rapidly for $k_{\theta} \dot{\mu}_{i} \geqslant 1$ (III $\geqslant 8$ ). The shape of the spectral distribution does not change much in the nonlinear stage except for the shift of the peak of the spectrum toward low $m$ numbers. This is due to the nonlinear excitation of 
convective cells which tend to coalesce into larger cells. $\left|E^{2}(n)\right|$, on the other hand, indicates a drastic change when the instability develops into a nonlinear regime. At earlier times, we see the spectrum is peaked at $n=1\left(k_{z}=2 \pi / L_{z}\right)$ and decreases rapidly with increasing $n$. This is quite reasonable because the growth rate for $\mathrm{n}=2$ and 3 are much smaller. The $\mathrm{n}=0$ modes, which may be called convective cell modes $\left(k_{z}=0, \omega=0\right)$, also grow as the drift instability grows and eventually they dominate over the drift instability. Note that this is not the ambipolar field ( $\mathrm{m}=$ $\mathrm{n}=0$ ). The excitation of convective cells is clearly seen in the power spectrum plots $\mathrm{P}_{\mathrm{mn}}(\omega)$ at $\mathrm{r} / \mathrm{a}=0.5$ where we see both drift modes $(n \neq 0, \omega \neq 0)$ and convective cells $(n=0$, $\omega=0)$. For $m=3$ modes, power spectrum indicates the amplitude of drift mode $(n=1)$ is much larger than the convective cells $(\mathrm{n}=0)$, while for $\mathrm{m}=1$, convective cells are much stronger than the drift mode.

The physical mechanism of the excitation of convective cells is the following. Consider a drift-wave fluctuation $\phi(r, \theta, z, t)=\phi_{m n}(r) \exp \left[i\left(\omega t-m \theta-k_{z} z\right)\right]$. Both ions and electrons will move through the $\mathrm{CE}_{\theta} / \mathrm{B}_{\mathrm{O}}$ drift in the radial direction. For a linearly unstable drift-wave $v_{i}<\omega / k_{z}$ $<v_{e}$ is satisfied so that the electron $\mathrm{CE}_{\theta} / \mathrm{B}_{0}$ drift tends to be averaged out on the time scale of drift wave because of the free streaming along the field lines. On the other hand, ions are essentially cold and respond to $\mathrm{cE}_{\theta} / \mathrm{B}_{\mathrm{O}}$ drift which 
in turn generates local charge separation and therefore a strong radial electric field. In fact the ion $\underset{\sim}{\mathrm{CE}} \times \underset{\sim}{\mathrm{B}}$ flows from $a$ two-dimensional vortex or convective cell as studied earlier in detail. 11 The number of convective cells is much more than that of unstable drift modes and the nonlinear interaction among convective cells can easily lead the plasma into a strong turbulence state due to very effective $\underset{\sim}{\mathrm{E}} \times \underset{\sim}{\mathrm{B} / \mathrm{B}^{2}} \mathrm{mixing}$. It is natural to expect $k_{\theta} \approx k_{r}$ for such a strong turbulent regime. Among the modes, due to nonlinear excitation, only the $\mathrm{k}_{\|}=0$ modes are important for anomalous plasma diffusion due to their long lives. ${ }^{11}$ It should be emphasized ${ }^{12}$ that the convective cells in a two-dimensional nonuniform plasma are linearly stable and have small amplitudes unless they are driven by some means such as drift instabilities in the present case for a collisionless plasma.

Figure 8 shows the formation of convective cells and ion diffusion due to nonlinear interactions among cells. Plotted are the guiding center orbits of the test ions initially sampled to form a ring at $r / a=0.5$. At early times, the test ions respond to $\mathrm{CE}_{\theta} / \mathrm{B}_{0}$ drift and one sees that several $m$ modes are excited. As the instability develops, ion motion becomes highly nonlinear and tends to form vortex structure as clearly seen in the figure. Small cells eventually coalesce into larger cells and the ions diffuse across the entire plasma column.

In order to confirm the importance of convective cells on plasma diffusion, we carried out another simulation with 
the same parameters except that the two-dimensional modes $\left(\mathrm{k}_{\mathrm{z}}=0\right)$ are articifially suppressed in the code. Figure 9 indicates the electron diffusion with and without $\mathrm{k}_{\mathrm{z}}=0$ modes. At early stage $\left(\omega_{p e} t=1000\right)$, diffusion is mainly due to drift instability and little difference is observed for both cases. At the later stage, however, diffusion without convective modes is substantialiy smaller than the other case and diffusion stops completely as the drift instability: saturates and the finite density gradient is maintained. For the other case, convective cells eventually flatten the density profile completely even when the drift instability has been quenched by nonlinear effects. This observation has an important significance in the understanding of anomalous diffusion in laboratory experiments. While the convective cells are marginally stable, ${ }^{12}$ drift or trapped-particle instabilities can excite the convective cells which lhen cause large diffusion even when the drift instabilities saturate nonlinearly. In a steady-state experiment, where the energy input such as that due to ohmic heating and particle recycling balances with the anomalous diffusion, it is likely that the diffusion due to drift instabilities is small and the steady state is established by balancing the energy source with the convective diffusion.

The observed diffusion coefficient is smaller than but not too far from the usual estimate of $D_{\perp}=\gamma / k_{\perp}^{2}$ for the initial diffusion due to drift instability and at a later stage, 
is also in good agreement with the convective cell diffusion formula when the enhanced level of fluctuations are used.

It is interesting to compare our results with recent laboratory experiments in toroidal devices. The recent FM-1 results ${ }^{8}$ indicate that for very strong shear $\left(L_{s} / L_{n}=7\right)$, the power spectrum shows a well-defined peak near the drift frequency. As the shear is reduced, a new peak appears near $\omega \approx 0$ and further reduction of shear results in a turbulent spectrum with no well-defined peaks. ${ }^{8}$ The two-peak spectrum shown in Fig. 6 resembles that of FM-1. A similar result is reported in the Culham Levitron experiment. 9

While our power spectrum is not as broad as those in the toroidal experiments with weak shear, the spectrum in the simulation is measured in a transient state from initial linear phase to the final turbulent state while the laboratory experiments measure fully developed steady-state turbulence driven externally. Furthermore, the spectrum in the laboratory experiments is averaged over different $k_{\|}$modes and the plasma radius. The simulation spectrum will be monotonic if such averaging is carried out.

Figure 10 shows the time development of the electric field energy associated with the drift wave $(n=1)$ and the convective modes $(n=0)$. We find eeveral drift modec ( $m=3,4$ and 5) grow to large amplitude with the expected growth rate in agreement with' the linear theory. The convective modes also grow at about twice the growth rate of the 
drift mode starting at a somewhat later time. This is an indication that the drift and convective modes are strongly coupled and the latter are driven unstable by the former.

Based on these observations, a theory is developed to show that the nonlinear interaction of drift waves can resonantly excite convective cells at the wavelength comparable to the ion gyroradius where drift waves are highly dispersive and the growth rate is the maximum. ${ }^{13}$ The observations are consistent with the theoretical considerations

We have simulated the same example keeping 11 modes in $z_{,}(n=0, \pm 1, \ldots ., \pm 5)$ and found the results essentially the same. We have also included a magnetic shear in the simulations and found the convective cells persist tor moderate strength of shear $\left(L_{s} / L_{n} \leqslant 25\right)$. 
VII. QUASI-NEUTRAL AND HYBRID PLASMA MODELS

In the preceding sections, three-dimensional plasma models in magnetic fields have been developed, tested and applied to plasma confinement studies. Except for the guiding center approximations for the electron transverse motion across magnetic field, full dynamics has been followed for ions and electrons. The highest frequency of the fluctuations in the model is therefore associated with the electron motion along the field lines whose characteristic frequency is given by $\left.\omega \sim \omega_{p e} k^{k}\right|^{k}$ or $\omega_{p i}{ }^{5}$ This frequency is substantially smaller than the electron plasma frequency for the case of $k_{\|} / k_{1} \ll 1$ and one can use $\omega_{p e} e^{\Delta t}$ considerably larger than 1 . On the other hand, when one is interested in low frequency phenomena such as drift or trapped particle instabilities or magnetohydrodynamic oscillations, it may be a good approximation to discard the high frequency oscillations associated 
with the electron inertia while keeping the ion dynamics and low frequency electron dynamics unmodified.

Whether or not it is possible to construct such a model depends on the problems considered. Furthermore whether or not such a model correctly simulates real plasma dynamics must be carefully examined for each case since the small departure from the charge neutrality gives rise to strong electric fields which modify particle dynamics.

Here we will briefly describe two different methods for eliminating high frequency space charge oscillations, one of which is a discrete particle model and the other particlefluid hybrid model. We first. consider a discrete plasma model and then discuss briefly the hybrid model.

In order to develop a particle simulation model which eliminates the high frequency oscillations, let us consider the simplest example first; namely, the one-dimensional ion sound wave in a thermal plasma. Assume a low frequency ion-density fluctuation $n_{i}^{\prime}$ from its equilibrium state $n_{0}$

$$
n_{i}=n_{0}+n_{i}^{\prime}:
$$

Associated with this density fluctuation is the low frequency electrostatic potential $\phi$ in which the electrons quickly reach the well-known Boltzmann; law

$$
\mathrm{n}_{\mathrm{e}}=\mathrm{n}_{\mathrm{o}} \exp \left(\mathrm{e} \phi / \mathrm{T}_{\mathrm{e}}\right)
$$


The resultant potential is then determined from the Poisson equation

$$
\partial^{2} \phi / \partial x^{2}=-4 \pi e\left(n_{i}-n_{e}\right)
$$

Ion-density fluctuation may be determined from the cold fluid equations

$$
\begin{aligned}
& \frac{\partial n_{i}}{\partial t}+\frac{\partial}{\partial x}\left(n_{i} v_{i}\right)=0 \\
& \frac{\partial v_{i}}{\partial t}+v_{i} \frac{\partial v_{i}}{\partial x}=-\frac{e}{m_{i}} \frac{\partial \phi}{\partial x} .
\end{aligned}
$$

Linearizing Eqs. (13) and (15) and assuming a phaser $\exp (i \omega t-i k x)$ for the linear quantities, density fluctuations for the electrons, the ions, and the dispersion relation are

$$
\begin{aligned}
& n_{e}^{\prime} / n_{0}=e \phi / T_{e} \\
& n_{i}^{\prime} / n_{0}=\left(k^{2} / \omega^{2}\right)\left(e / m_{i}\right) \phi
\end{aligned}
$$

and

$$
\begin{aligned}
\left(k^{2}+k_{e}^{2}\right) \phi & =4 \pi e_{i}^{\prime} \\
& =\left(k^{2} \omega_{p i}^{2} / \omega^{2}\right) \phi
\end{aligned}
$$


where $k_{e}$ is the electron Debye wave number, $k_{e}^{2}=4 \pi n_{0} e^{2} / T_{e}$. Equation (18) gives the well-known dispersion relation for the ion wave

$$
\omega^{2}=\mathrm{k}^{2} \mathrm{c}_{\mathrm{s}}^{2}=\mathrm{k}^{2} \mathrm{~T}_{\mathrm{e}} / \mathrm{m}_{\mathrm{i}}
$$

for $\mathrm{k}^{2} \ll \mathrm{k}_{\mathrm{e}}^{2}$. When the dispersion relation is used in Eqs. (16) and (17), it is easy to show that the quasineutrality $n_{e}^{\prime}=n_{i}^{\prime}$ is satisfied automatically.

It is clear in the preceding argument that the key assumption for the charge neutrality is given by Eq. (13) or Eq. (16) where the massless electrons follow the ions adiabatically so as to screen the resultant potential. Note that the quasineutrality $n_{i}^{\prime}=n_{e}^{\prime}$ is the consequence of Eq. (16). The effect of electrons can be seen more clearly from Eq. (18) where the potential due to Ion-densily fluctuation is Debyescreened by the electrons and this is the physical interpietation of the quasineutrality.

In the usual particle simulation model, both iuns and electrons are pushed according to the equation of motion together with the Poisson equation to determine the potential. In such a model, it is clear that the charge neuliality, Eq. (16), is not satisfied in general, since the electrons do not always follow the ions because of their inertia. In fact, it is well known that the electron and ion density fluctuations are predominantly out of phase, which generate 
very strong electrostatic space charge oscillations at the plasma frequency. This high frequency oscillation is so strong in numerical simulations that it can mask the weak ion sound wave easily for many cases.

It is clear, from the preceding argument, that one way to guarantee the quasineutrality in the simulation is to assume that the electrons form a Deby-shielding cloud around the ion-density fluctuations. The Poisson equation is then modified to

$$
\left(k^{2}+k_{e}^{2}\right) \phi=4 \pi e\left(n_{i}-\dot{n}_{o}\right)
$$

together with the equation of motion for the ions

$$
\begin{aligned}
\frac{d v_{i}}{d t} & =\frac{e}{m_{i}} E \\
\frac{d x_{i}}{d t} & =v_{i}
\end{aligned}
$$

to determine the ion density. Note that in this formalism, the contribution from the electrons is given totally by $\mathrm{k}_{\mathrm{e}}^{2}$, which is valid as long as they are adiabatic. When the contribution of the nonadiabatic electrons becomes important, then Eq. (19) should be modified accordingly. This is a more difficult problem and the solution appears to depend on the problems considered. 
Note also that there is no approximation made for the ion dynamics in the model so that the model should be very useful to study the nonlinear behavior of plasmas associated with ion dynamics.

The linearization of the electron Boltzmann distribution used in Eq. (19) is valid as long as the density fluctuation $n_{i}^{\prime} / n_{0}$ is a few percent. When $n_{i} / n_{0}$ becomes larger, then the linearization is not appropriate and one has to use the nonlinear Poisson equation given by

$$
\nabla^{2} \phi=4 \pi e n_{i}-4 \pi e n_{o} \exp \left(e \phi / T_{e}\right)
$$

It is possible to solve this by iteration for this case. First, the solution for the potential $\phi_{\Omega^{\prime}}$ using the linearized density fluctuations, is found from Eq. (19). Then the corrected potential $\phi$ is tound bỵ using $\phi_{0}$ in lle eleclium Boltzman distribution to solve for $\phi$. One can iterate this procedure a few times until the solution converges.

So far we have only ronsidered the one-dimensional fon sound wave. It is clear that the model can be extended to many different cases in multi-dimensional simulations as long as the electron contribution is primarily adiabat1c, which is the characteristic property for low frequency oscillations. The electron density fluctuation for the electrostatic waves in a magnetic field may be written as, in general, 14 


$$
\begin{aligned}
4 \pi e_{e}^{\prime}=k_{e}^{2} & \sum_{n=-\infty}^{\infty} \exp \left(-k_{y}^{2} v_{e}^{2} / \Omega_{e}^{2}\right) I_{n}\left(k_{y}^{2} v_{e}^{2} / \Omega_{e}^{2}\right) \\
\cdot & {\left[1+\frac{(1)-(1)_{e}^{*}}{\sqrt{2 k_{z} v e}} Z \cdot\left(\frac{(\omega)-n \cdot \Omega_{e}}{\sqrt{2 k_{z} v_{e}}}\right)\right] }
\end{aligned}
$$

where the magnetic field is in the $z$-direction, $\omega^{*}=\left(\mathrm{CT}_{\mathrm{e}} \mathrm{k}_{\mathrm{y}} / \mathrm{eB}\right)$ ( $d$ ln $n / d x$ ) is the diamagnetic drift frequency due to plasma inhomogeneity in the $\mathrm{x}$-direction, $\mathrm{z}$ is the plasma dispersion function, ${ }^{15}$ and the electron distribution function is assumed to be an isotropic Maxwellian.

For low-frequency $\omega \ll \omega_{c e}$, long wavelength $k_{y}^{2} v_{e}^{2} / \Omega_{e}^{2} \ll 1$ oscillations and $\mathrm{k}_{\mathrm{z}} \neq 0, \mathrm{Eq} \cdot(20)$ is reduced to

$$
4 \text { " e } \mathrm{n}_{\mathrm{e}}^{\prime}=\left[1+\frac{(1)-(1)^{*}}{\sqrt{2 \mathrm{k}_{\mathrm{z}} \mathrm{v}_{\mathrm{e}}}} \mathrm{Z}\left(\frac{(1)}{\sqrt{2} \mathrm{k}_{\mathrm{z}} \mathrm{v}_{\mathrm{e}}}\right)\right] \mathrm{k}_{\mathrm{e}}^{2} \phi
$$

For the small phase velocity $\omega / \sqrt{2} \mathrm{k}_{\mathrm{z}} \mathrm{v}_{\mathrm{e}} \ll 1, \mathrm{Eq} \cdot$ (21) reduces to Eq. (16); and therefore, Eq. (19) should be valid as long as the nonadiabatic effect is ignored.

Needless to say, there are many cases where the small non-adiabatic effect arising from the Landau or collisional damping can cause instabilities, in which case, those nonadiabatic electrons should be included in the simulation. Note also, nowhere have we assumed $n_{e}=n_{i}$ explicitly in the model, and this is very important, since $\mathrm{n}_{\mathrm{e}}$ is not exactly equal to $n_{i}$ for the unstable plasmas when $\phi$ grows to large amplitude. Such an example is the case of anomalous diffusion due to drift instabilities. 
Two examples of the model are given in the following. One of them is the propagation of the ion sound wave and the other is the fluctuations in a magnetic field.

1. Propagation of the Ion Sound Wave

In this first example, we have considered the propagation of ion sound waves in a thermal plasma and confirmed that the model correctly produces the expected ion sound fluctuations. The simulation is carried out in one dimension using an electrostatic dipole code. As described in the previous section, only the ions are pushed and the modified Poisson equation (19) is used.

Figure 11 shows the results from such a run. We use $\mathrm{T}_{\mathrm{e}} / \mathrm{T}_{\mathrm{i}}=10, \mathrm{~m}_{\mathrm{i}} / \mathrm{m}_{\mathrm{e}}=100, \omega_{\mathrm{pe}} \Delta \mathrm{t}^{\prime}=2$ ( $\Delta \mathrm{t}$ is the integration time step), $\lambda_{e}=\Delta=a$ ( $\Delta$ is the grid size and a is the particle size) and 6400 ions. Figure $11(a)$ shows the time correlation function of the fourth Fourier mode and Fig. $11(b)$ is the power spectrum of the same mode. We see a coherent wave at the expected frequency: of the ion sound wave. Note that there is no electron plasma oscillation in the model and the electric field energy is less than $10^{-4}$ of the particle energy. The two peaks in Fig. $\perp \perp(b)$ correspond to the sound wave propagating both directions in space.

Figure $12(\mathrm{a})$ shows the numerically determined dispersion relation for the above example. Nute the agreement with the theory 


$$
\omega_{s}=\frac{k c_{s}\left(1+3 T_{i} / 2 T_{e}\right)}{\left(1+k^{2} \lambda^{2} e^{k^{2} a^{2}}\right) 1 / 2}
$$

is excellent. Figure $12(\mathrm{~b})$ indicates similar results from the second run using $\omega_{p e} \Delta t=10\left(\omega_{p i} \Delta t=1\right)$ and $\lambda_{e}=0.2 \Delta$ with the other parameters unchanged. This run is to confirm that large time step, such as $\omega_{s} \Delta t \approx 0.2$ and small Debye length $\lambda_{e}<\Delta$, may be used in the model since there is no plasma oscillation in the model.

It is clear from these runs that the plasma oscillations are completely eliminated and that one should be able to use $\Delta t$ several orders of magnitude greater than that employed in the conventional code when realistic parameters are used. Note that there was no grid instability ${ }^{16}$ observed for small Debye length.

\section{Fluctuations in a Magnetic Field}

Let us consider the propagation of low-frequency ion fluctuations in a magnetic field. When an external magnetic tield is imposed on a plasma, motions across the magnetic field are prohibited and the quasineutrality is maintained as the electrons follow the ions along the field lines. It is clear then that the flute-typpe mode, $k_{z}=0$, for example, may not be quasineutral. Furthermore, whether or not the plasma is quasineutral in a magnetic field is not a trivial question in an inhomogeneous plasma where the low frequency turbulence generated by the drift-type instabilities causes 
the anomalous diffusion of ions and electrons which are in general not the same and, therefore, charge separations are built up to maintain the ambipolarity. 13

The electrostatic dispersion relation for small amplitude ion oscillations in a magnetic field is given by

$1+\frac{1}{k^{2} \lambda_{e}^{2}}+\frac{1}{k^{2} \lambda_{i}^{2}} \sum_{n=-\infty}^{\infty}\left[1+\exp (-\lambda) I_{n}(\lambda) \frac{(1)-(1)}{\sqrt{2 k v_{i}^{*}}} Z\left(\frac{()-n \Omega_{i}}{\sqrt{2 k v_{i}}}\right)\right]=0$

for $\mathrm{k}_{\mathrm{y}}^{2} \rho_{\mathrm{e}}^{2} \ll 1$ and $\omega / \mathrm{k}_{\mathrm{z}} \mathrm{v}_{\mathrm{e}} \ll 1$ where $\lambda=\mathrm{k}_{\mathrm{y}}^{2} \mathrm{v}_{\mathrm{i}}^{2} / \Omega_{\mathrm{i}}^{2}$. The interesting case is for $k_{z} / k_{Y} \ll 1$ and the solutions of Eq. (22) are well-known electrostatic ion-cyclotron waves (ion Bernstein modes)
$\omega=n s_{i}\left(1+I_{n}(\lambda) e^{-\lambda}\right)$
$11-1 ; 2, \ldots$.

and the low frequency ion acoustic mode

$$
w=k_{z} c_{s}
$$

and the drift mode

$$
\omega=\omega_{c}^{*}
$$

in an inhomogeneous plasma. Note that the lower hybrid oscillations $\omega \approx \omega_{\mathrm{pi}}$ and the oblique electron plasma osc1llations $\omega=\omega_{p e} k_{z} / k$ are not the solutions of Eq. (22). since 
they are shielded by the electrons.

To test the dispersion relation, a frequency spectrum for the fluctuations is measured using a 2 1/2-dimensional model ${ }^{5}$ with the modified Poisson equation (19). The parameters for the simulations are: 64 x 64 grid, 128 x 128 ions, $\mathrm{m}_{\mathrm{i}} / \mathrm{m}_{\mathrm{e}}=2500, \Omega_{\mathrm{e}} / \omega_{\mathrm{pe}}=5, \mathrm{~T}_{\mathrm{e}} / \mathrm{T}_{\mathrm{i}}=4, \lambda_{\mathrm{e}} / \Delta=1, \omega_{\mathrm{pe}} \Delta t=50$ and $k_{z} / k_{y}=\left(m_{e} / m_{i}\right)^{1 / 2}=0.02$. Note that the choice of the time step gives $\Omega_{i} \Delta t=0.1$, which is small enough to resolve cyclotron harmonics. Note also the large mass ratio and the time step in the present simulation.

Figure 13 shows the power spectrum for the propagation of different values of $k_{z} / k_{y}$ and $k_{y}$ in a homogeneous plasma. It is clearly seen that several ion-cyclotron harmonics are generated slightly above the cyclotron frequency and its harmorics. For $k_{1} \rho_{i} \leqslant 1$, the maximum peak of the spectrum is at the cyclotron frequency and it shifts to higher harmonics for $k_{\perp} \rho_{i}>1$. There is also one low frequency oscillation well below the ion-cyclotron frequency representing the low frequency ion sound branch. These frequencies agree well with the linear theory. The field energy was only $0.1 \%$ of the ion kinetic energy in spite of the use of only four particles per cell. Since the electrostatic ion-cyclotron harmonics are correctly simulated in this model, one can use the model, for example, to study the ion beam-induced cyclotron instabilities associated with the neutral injection into a tokamak. 17

Figure 14 shows the power spectrum of the fluctuations in an inhomogeneous plasma using the 2 1/2-dimensional model. 
In addition to the ion-cyclotron harmonics, low frequency drift oscillations can be seen clearly in the figure. The observed frequency is larger than the ion sound frequency in a homogeneous plasma shown in Fig. 13 and is close to $\omega=\omega_{e}^{*} \beta /(2-\beta)$ as predicted from the linear theory where $\beta=e^{-\lambda} I_{0}(\lambda)$. The parameters of the simulation are the same as before except for the inhomogeneous density profile' in the $x$-direction, which is taken as a hyperbolic tangent. 5 The observation of low frequency drift oscillations $\omega \ll \Omega_{i}$ using realistic plasma parameters is quite ençouraging and the model may serve as an important tool for the study of nonlinear development and the anomalous diffusion due to drift instabilities in threedimensional cylindrical and toroidal simulations. 3 These calculations using the 2 1/2-dimensional model took one half hour or so on IBM $360 / 91$ for each case.

We shall not discuss here how to include the nonadiabatic electrons due to collisionless Landau damping or collisional effects, although they are apparently important for the specific problems considered. Generally speaking, how to treat the nonadiabatic electrons is a subtle problem and appears to depend on the cases considered. Some examples can be tound in lief. 18 .

We shall now briefly discuss the hybrid approach to plasma simulation, namely, fluid electrons and discrete particle ions. In this model, the electrons are assumed some sort of resistive fluid obeying the generalized ohm's 
law. Quasi-neutrality is often assumed $\left(\mathrm{n}_{e}=\mathrm{n}_{i}\right)$ and the electron inertia is neglected to disregard the high frequency oscillations. 19,20

obviously the validity of the model has a number of restrictions. First of all, the electrons must be in a collisional regime in which the mean free path is shorter than the wavelength of interest and the collision frequency larger than the characteristic frequency. For tokomak plasmas which are low density and high temperature, these conditions are likely not to be met. On the other hand, plasmas in pinch devices are in higher density and lower electron temperature and therefore it may be pretty safe for the use of electron fluid model even though amonalous transport coefficients are often used to get reasonable agreement between simulation and laboratory experiments, 19,20

When the plasma density in a tokamak becomes much higher than the present one, a particle-fluid hybrid model may be used. Even for present-day tokamak, it will be a powerful tool to study phenomena such as resistive tearing mode instabilities where the kinetic effects of the ions can be conveniently included by the discrete particle models.

\section{CONCLUSIONS}

We have developed three-dimensional plasma models for cylindrical and toroidal systems by employing the combination of eigenfunction expansion in one direction and the multipole 
expansion using a two-dimensional spatial grid. Tests of the models correctly reproduce the expected fluctuation spectrum for a plasma near thermal equilibrium. Application of the model to the anomalous diffusion due to collisionless drift instability is given.

Furthermore some considerations are given for the quasineutral particle model and the particle-fluid hybrid model.

The impact on plasma physics and controlled fusion from the present day computer simulations is already significant and it is clear that the numerical simulation approach will be essential in both nonlinear plasma theory and fusion applications in the near future when larger and faster computers will become available.

\section{ACKNOWLEDGEMENTS}

'The author is grateful to' the members of the Plasma Physics Laboratory for the support of this work and for useful. discussions. Particular thanks are due to Dr. C. Z. Cheng, Dr. W. W. Lee, Dr. A. T. Lin and Professor J. M. Dawson. This work is supported by the United States Energy Research and Development Administration Contract E(11-1) - 3073 . 


\section{REFERENCES}

1 o. Buneman, Phys. Rev. 115, 503 (1959).

2 J. M. Dawson, Phys. Fluids 5,445 (1962).

3 C. Z. Cheng and H. Okuda, J. Compt. Phys. (To appear)

Princeton Plasma Physics Laboratory Report MATT-1204

4 A. B. Langdon, J. Compt. Phys. 6, 247 (1970).

5 W. W. Lee and H. Okuda, J. Compt. Phys. (To appear)

Princeton Plasma Physics Laboratory Report MATT-1231

6 E. Mazzucato, Phys. Rev. Lett. 36, 792 (1976).

7 S. M. Hámberger, I. E. Sharp, J. B. Lister and S. Phys. Rev. Lett. 37,1345 (1976).

8 M. Okabayashi and V. Arunesalum, Princeton Plasma Physics Laboratory Report PPPL-1310 (1977).

9 M. W. Alcock, D. E. T. F: Ashby, J. G. Gordey, T. Edlington, W. H. W. Fletcher, E. M. James, J. Malmberg; A. C. Rivier, D. F. H. Start and D. R. Sweetman, IAEA 6th Conference on Plasma Physics and Controlled Fusion, CN 35/DI2 (1976)

${ }^{10}$ W. W. Lee and H. Okuda, Phys. Rev. Lett. $\underline{12}, 870$ (1976).

$11_{\mathrm{H}}$. Okuda and J. M. Dawson, Phys. Fluids 16, 408 (1973).

${ }^{12} \mathrm{~J}$. Canosa, J. Krommes, C. Oberman, H. Okuda, K. Tsang, J.M. Dawson and T. Kamimura, IAEA 5 th Conference on Plasma Physics and Controlled Fusion, Paper CN 33/J2, p. 177 (Vienna 1975). 
${ }^{13} \mathrm{H}$. Okuda and C. Z. Cheng, Princeton Plasma Physics Laboratory Report PPPL-1328, 1316.

${ }^{14}$ A. Hasegawa, Phys. Rev. 169, 204. (1968).

${ }^{15}$ B. D. Fried and S. D. Conte, The Plasma Dispersion Function, (Academic Press, New York 1961).

16 L. Chen and H. Oḳuda, J. Compt. Phys. 19, 339 (1975).

${ }^{17}$ H. W. Hendel, M. Yamada, S. W. Seiler and H. Ikezi, Phys. Rev. Letters 36, 319 (1976).

${ }^{18}$ H. okuda, J. M. Dawson, A. T, T.in and r. r. Tin, Phys. Fluids (To appear). Princeton Plasma Physics Laboratory Report PPPL-1317.

${ }^{19}$ R. Chodura, Nucl. Fusion 15, 55 (1975).

20 A. G. Sgro and C. W. Nielson, Phys. Fluids 19, 126 (1976). 


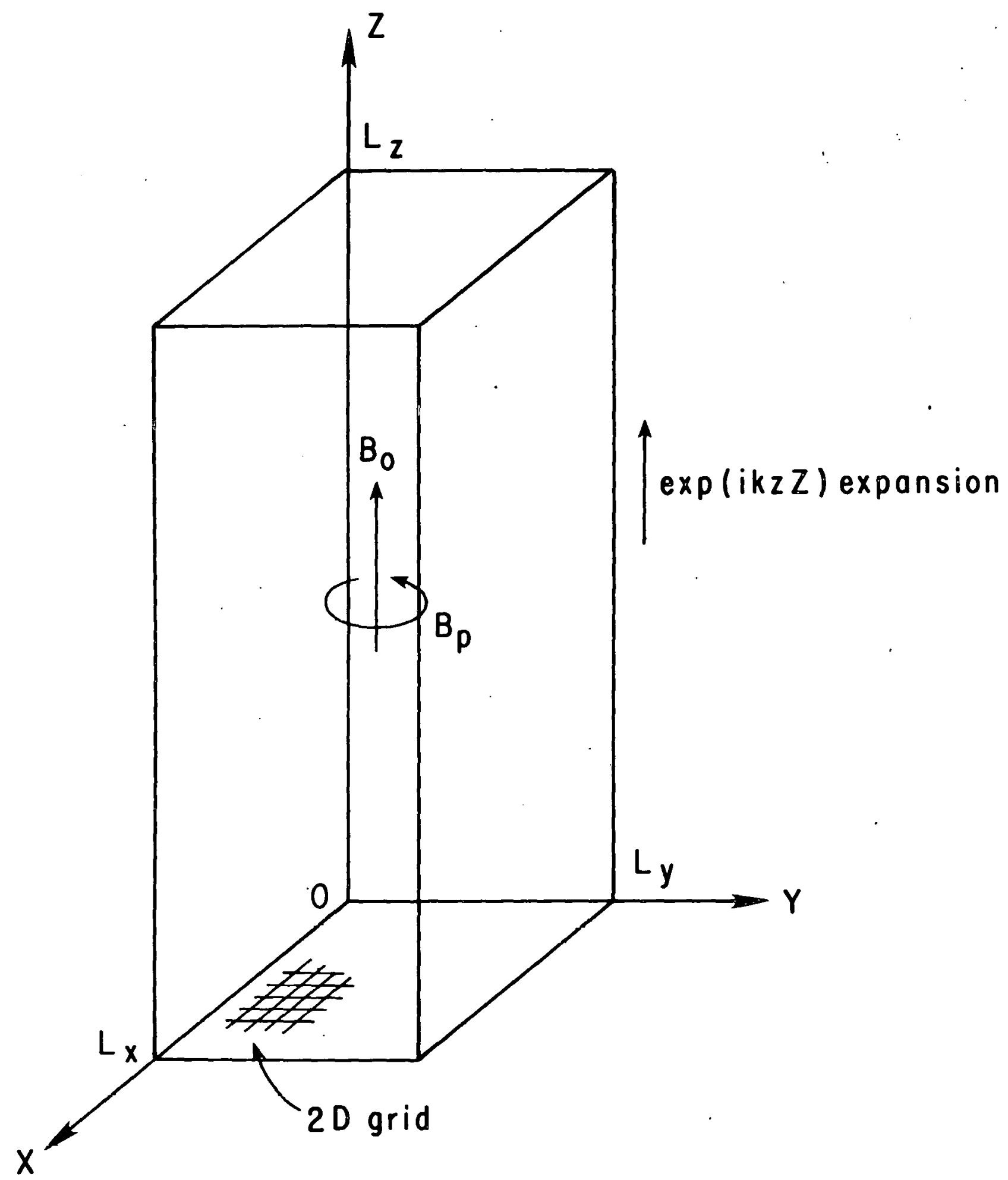

772047

Fig. 1. A sketch of the three-dimensional model. Twodimensional spatial grid is used in $(x, y)$ plane and the expansion in terms of Fourier series is used in the z-direction. 


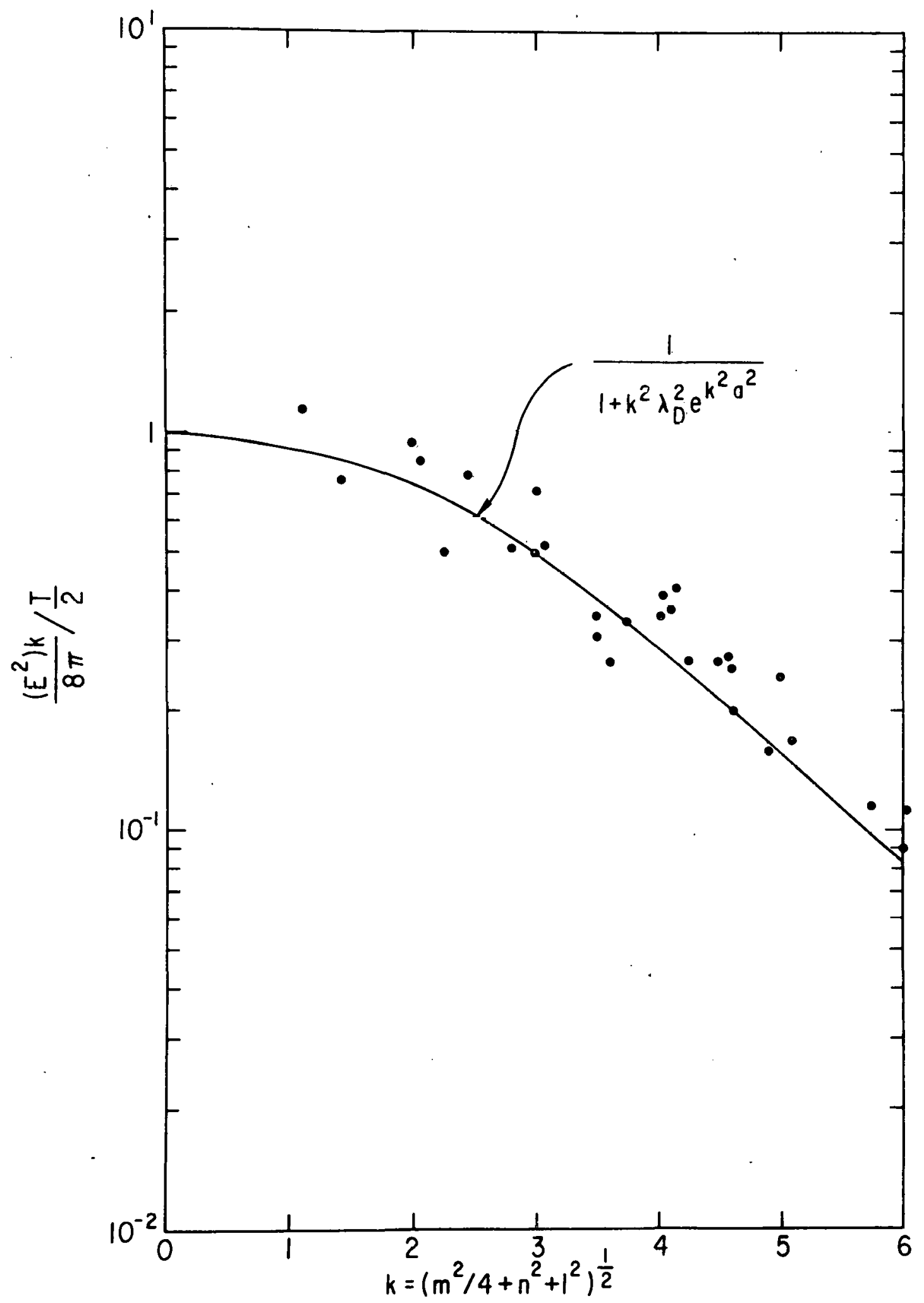

762093

Fig. 2. Time averaged fluctuation spectrum of the electric field energy for a mode $\underset{\sim}{k}=\left(2 \pi m / L_{x}, 2 \pi n / L_{y}, 2 \pi l / L_{z}\right)$. Agreement between the measurement and the theoretical prediction is good. 


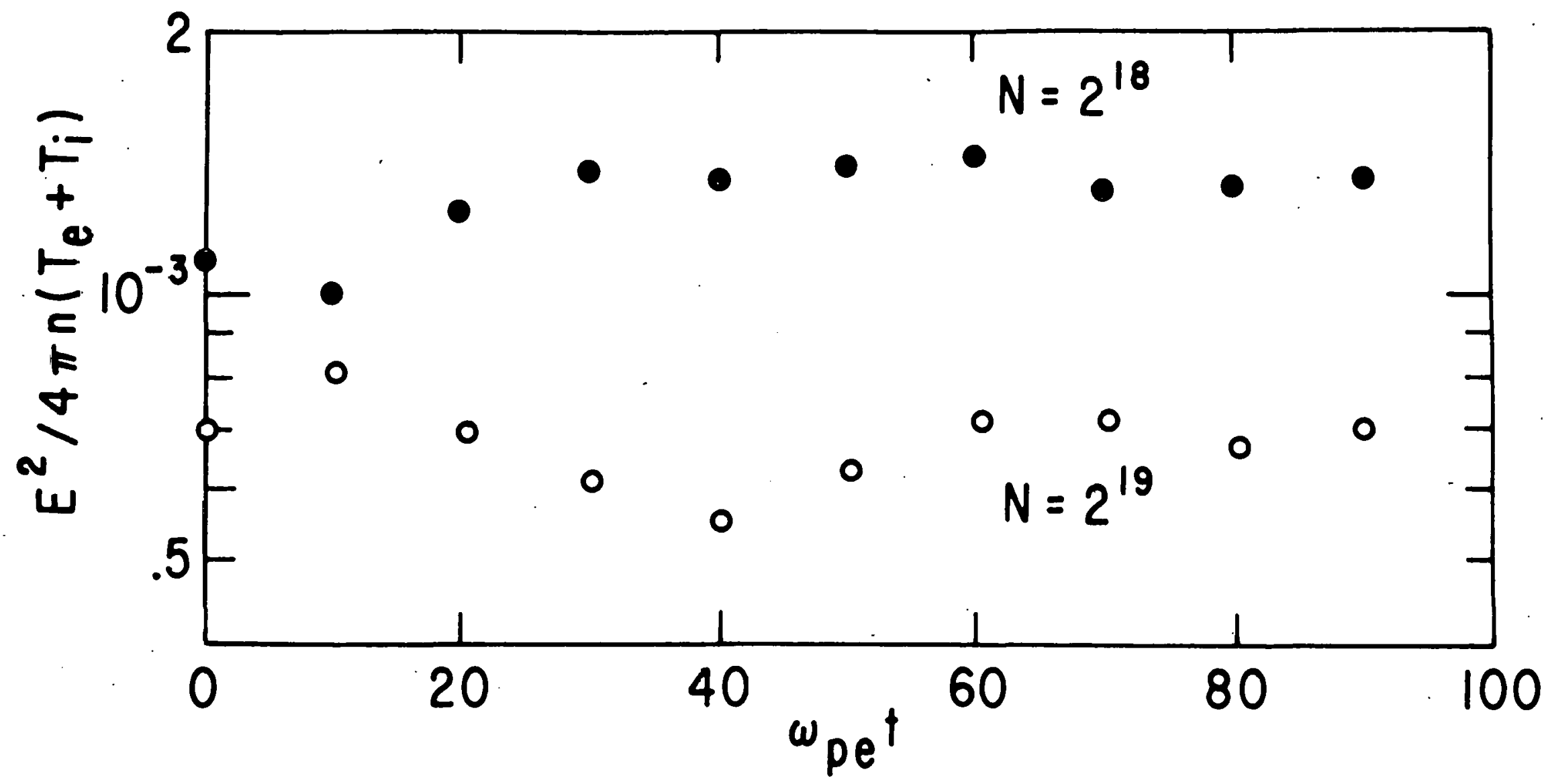

772046

Fig. 3. Random thermal fluctuations of the field energy normalized by the kinetic energy. The noise level is $10^{-4} \mathrm{n}$ $10^{-3}$ of the kinetic energy when the total number of simulation particles is $10^{5} \sim 10^{6}$. 
$\stackrel{1}{\infty}$
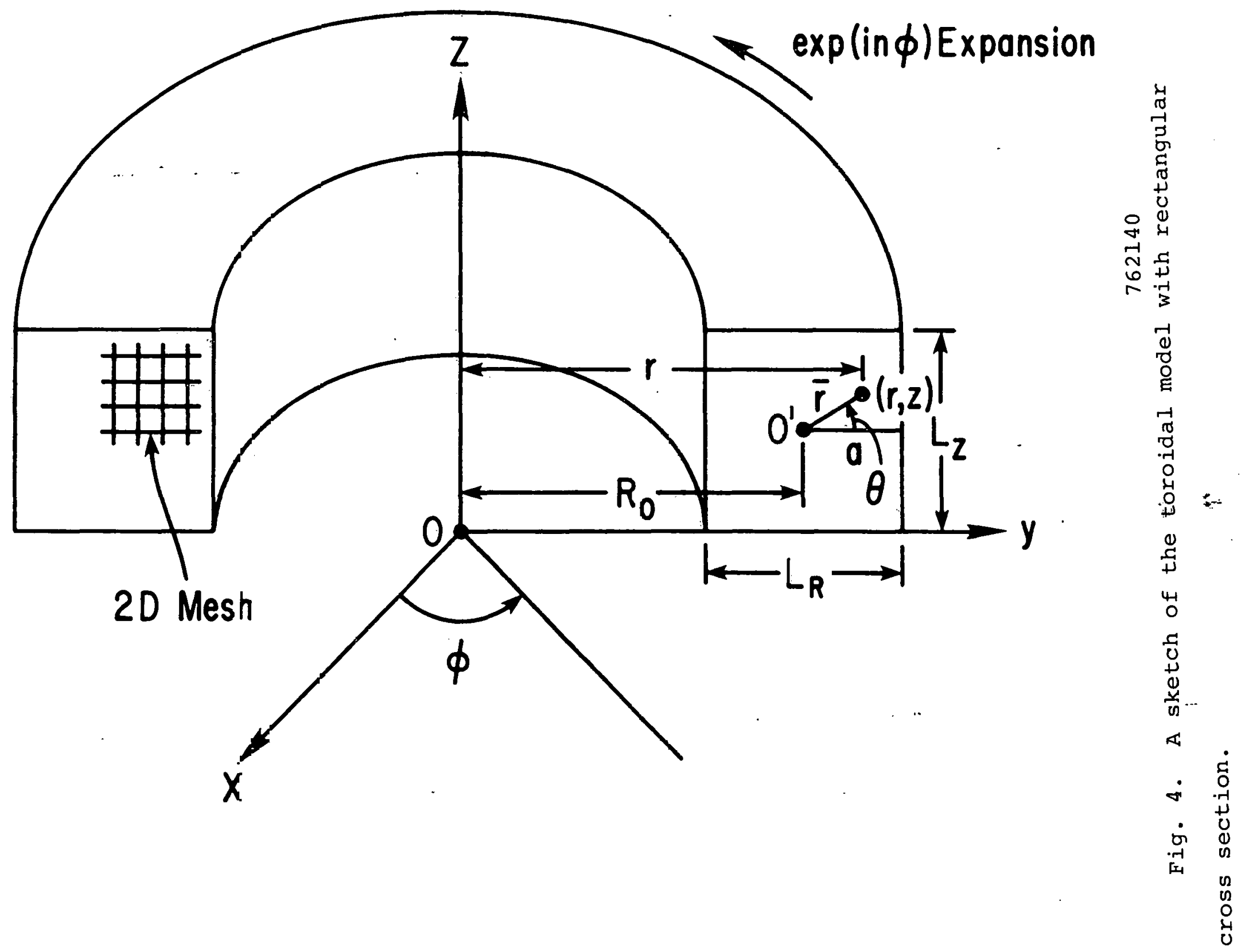


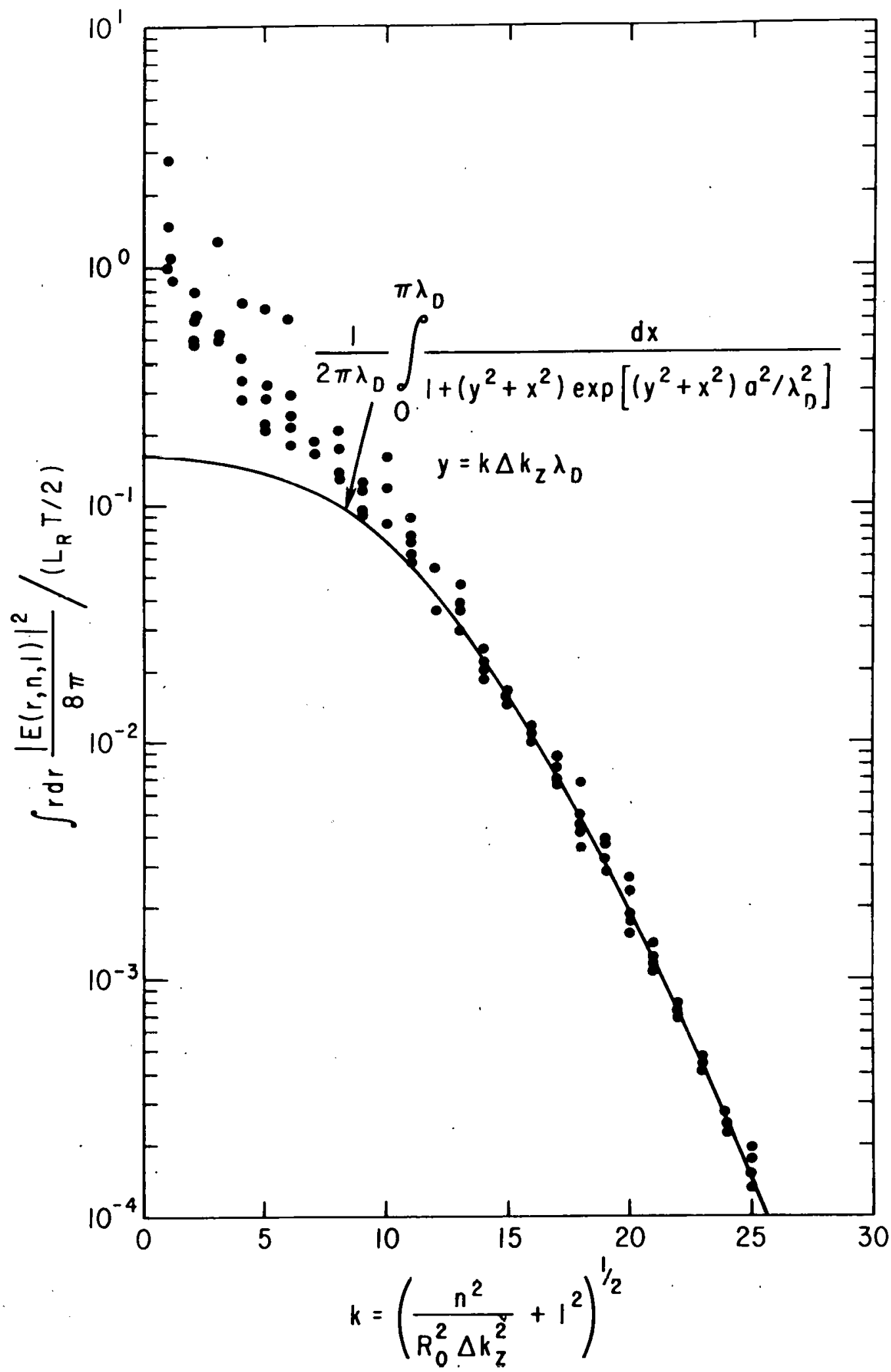

Fig. 5. Fluctuation spectrum averaged over the radial direction for the toroidal model. The enhancement at small k's is due to $\nabla B$ drift of the ions. 

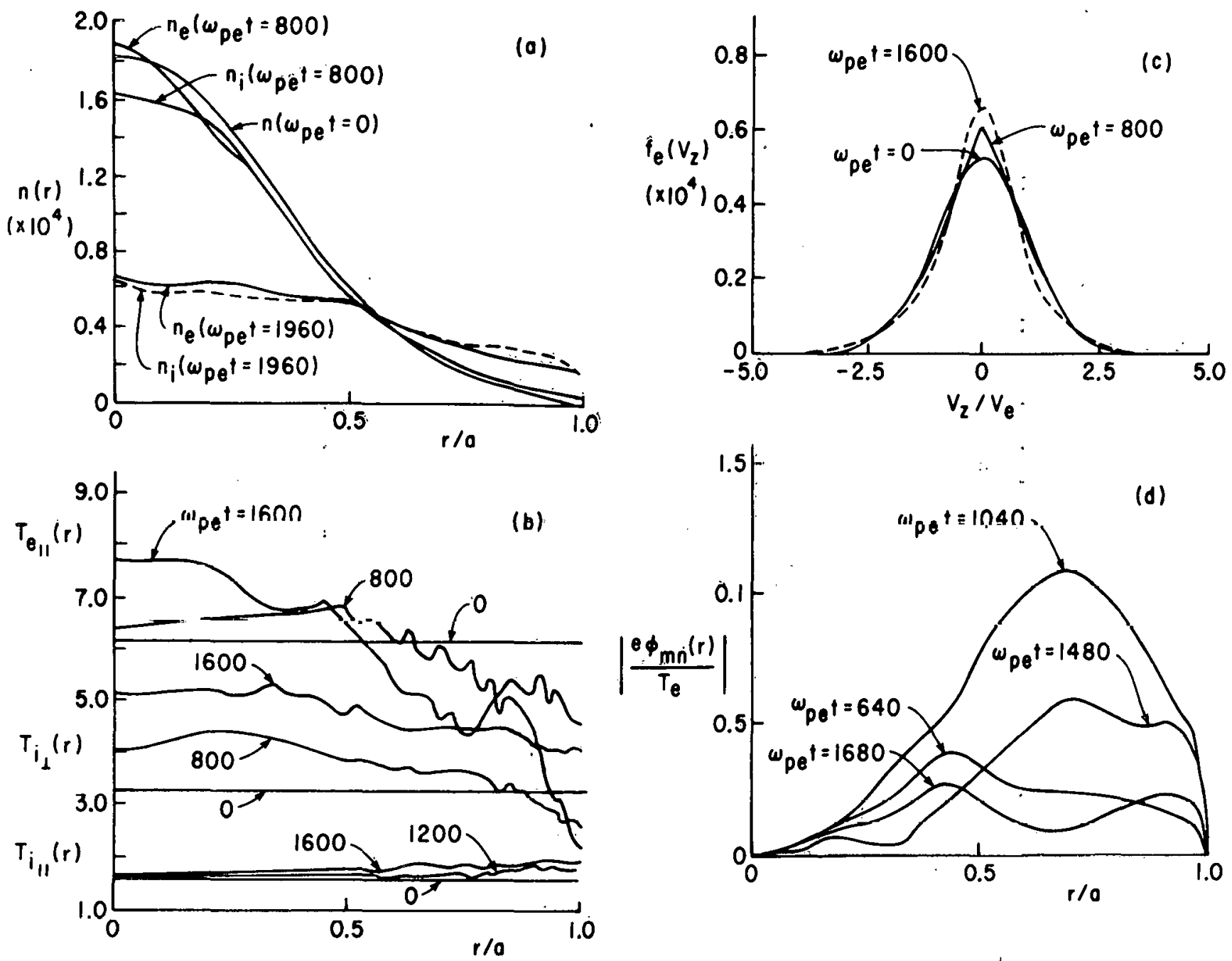

Fig. 6. Time variation of (a) ion and electron density profiles $n_{i}(r), n_{e}(r)$. Note the generation of charge separation in the later stage, (b) electron parallel temperature $\mathrm{T}_{\mathrm{e}} \|(\mathrm{r})$, ion temperatures $\mathrm{T}_{i \|}(r)$ and $\mathrm{T}_{\mathrm{I}^{\perp}}(\mathrm{r}),(\mathrm{c})$ electron velocity distribution, and (d) radial mode structures for $(m, n)=(3,1)$ mode. 

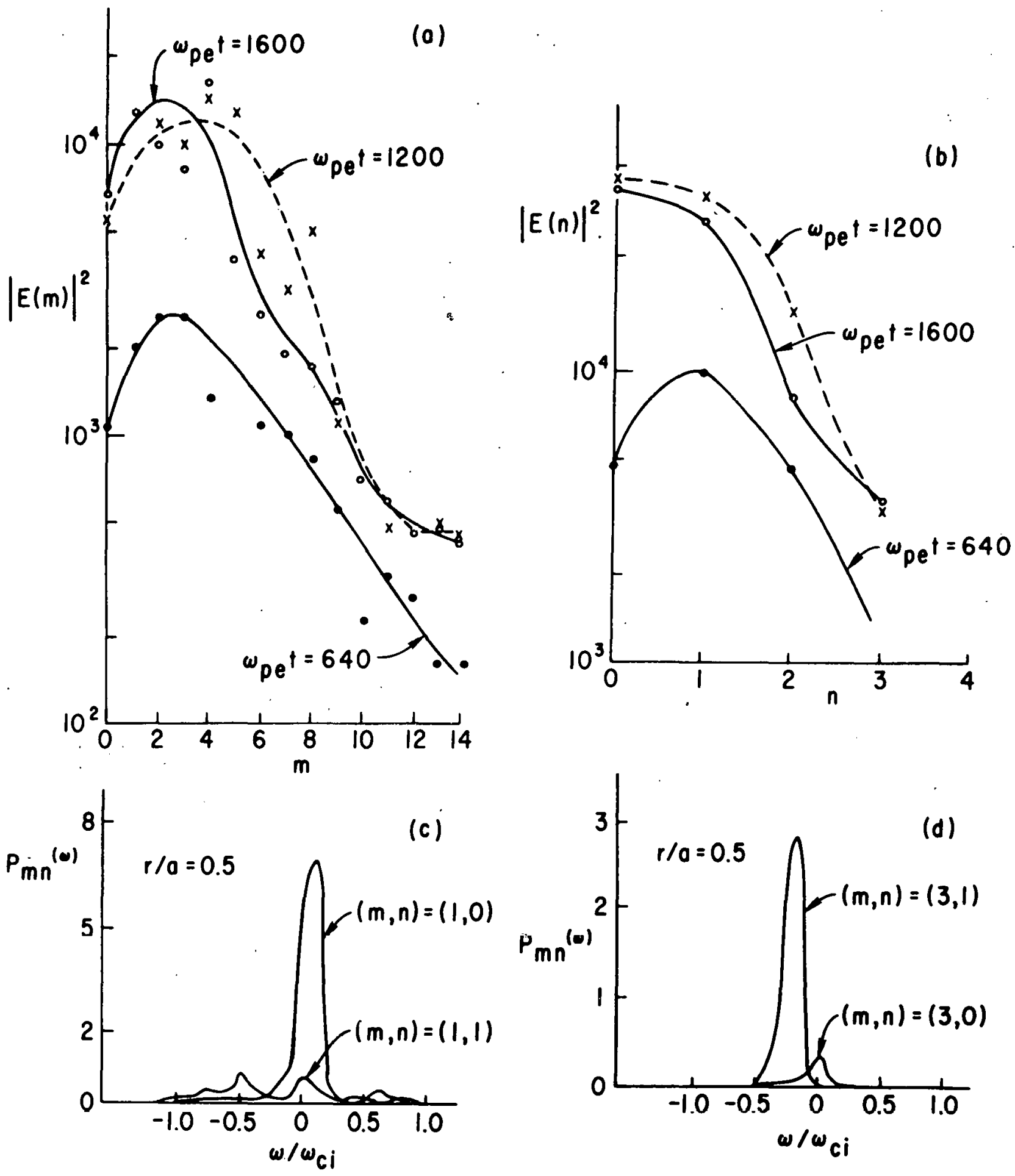

762387

Fig. 7. Time variation of (a) spectral distribution $\left|E^{2}(m)\right|$ and (b) epoatral dictribution $\left|E^{2}(n)\right|$. Power spcetra for $(c)(m, n)=(1,1),(1,0)$ modes and for $(d)(m, n)=(3,1)$, $(3,0)$ modes indicate two peaks near $\omega \approx 0$ and $\omega^{\star}$. 


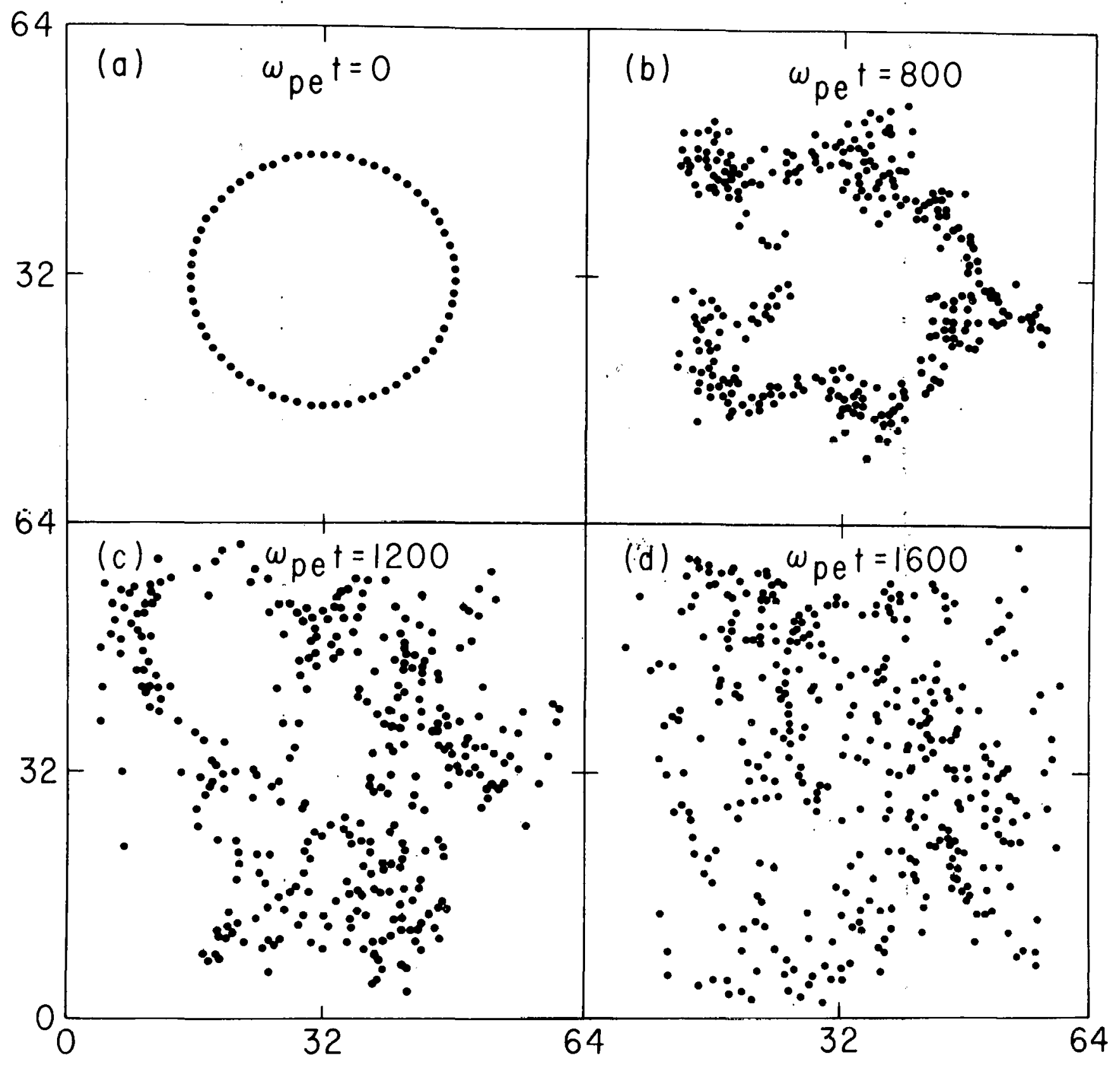

762383

Fig. 8. Plot of the guiding center orbits of test ions initially sampled to form a ring at some fixed $z$. Note the development of convective cells and the coalescence into larger cells. 


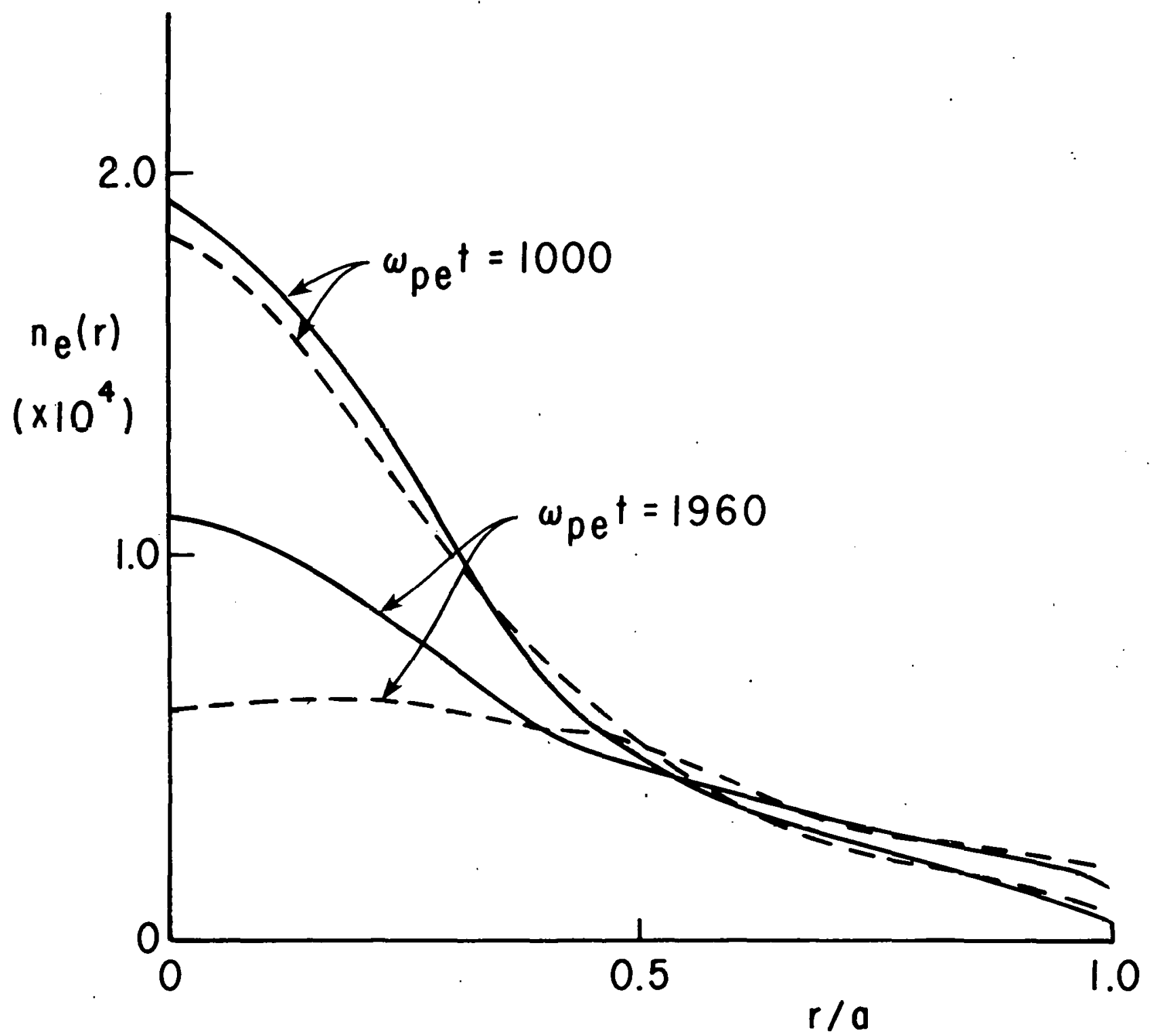

762384

Fig. 9. Comparison of electron density diffusion with and without convective cells $\left(k_{\|}=0\right)$. 

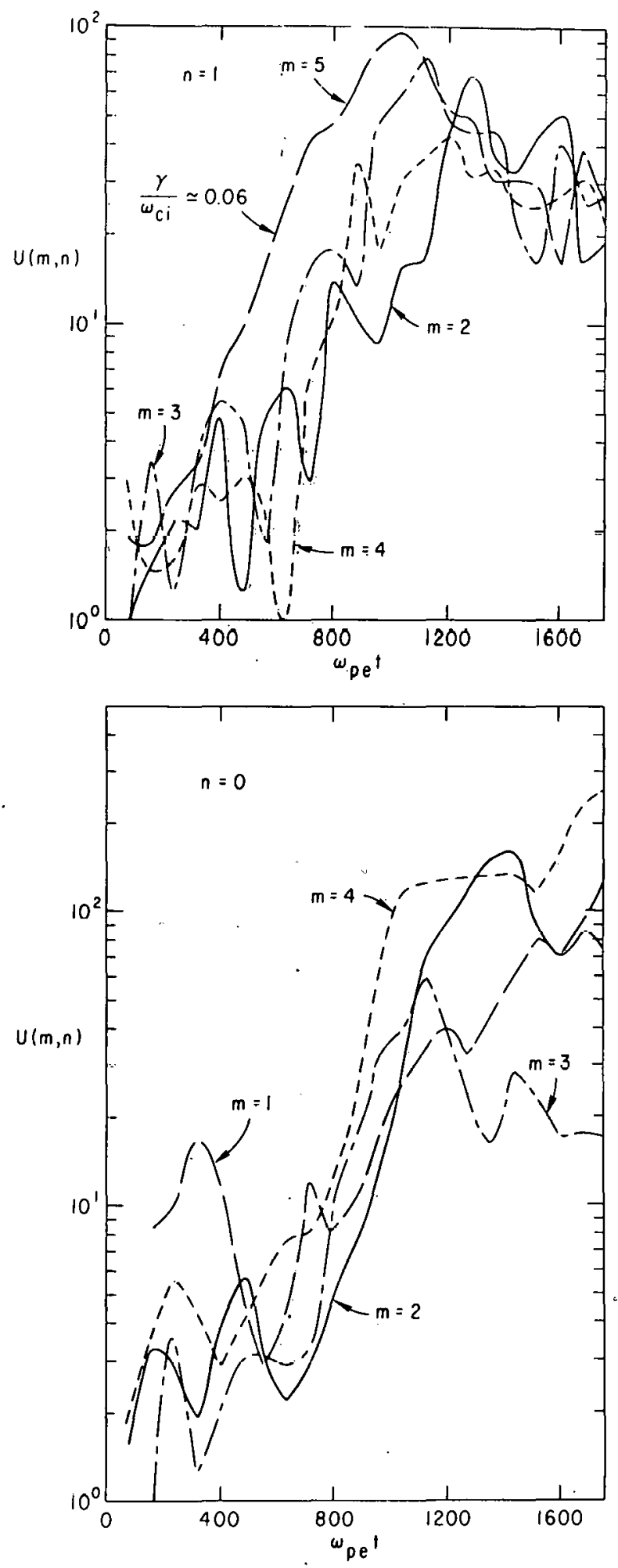

772013

Fig. 10. Time development of the drift modes $(n=1)$ and convective cells $(n=0)$. Note the convective cells grow faster than the drift waves starting somewhat later time. 

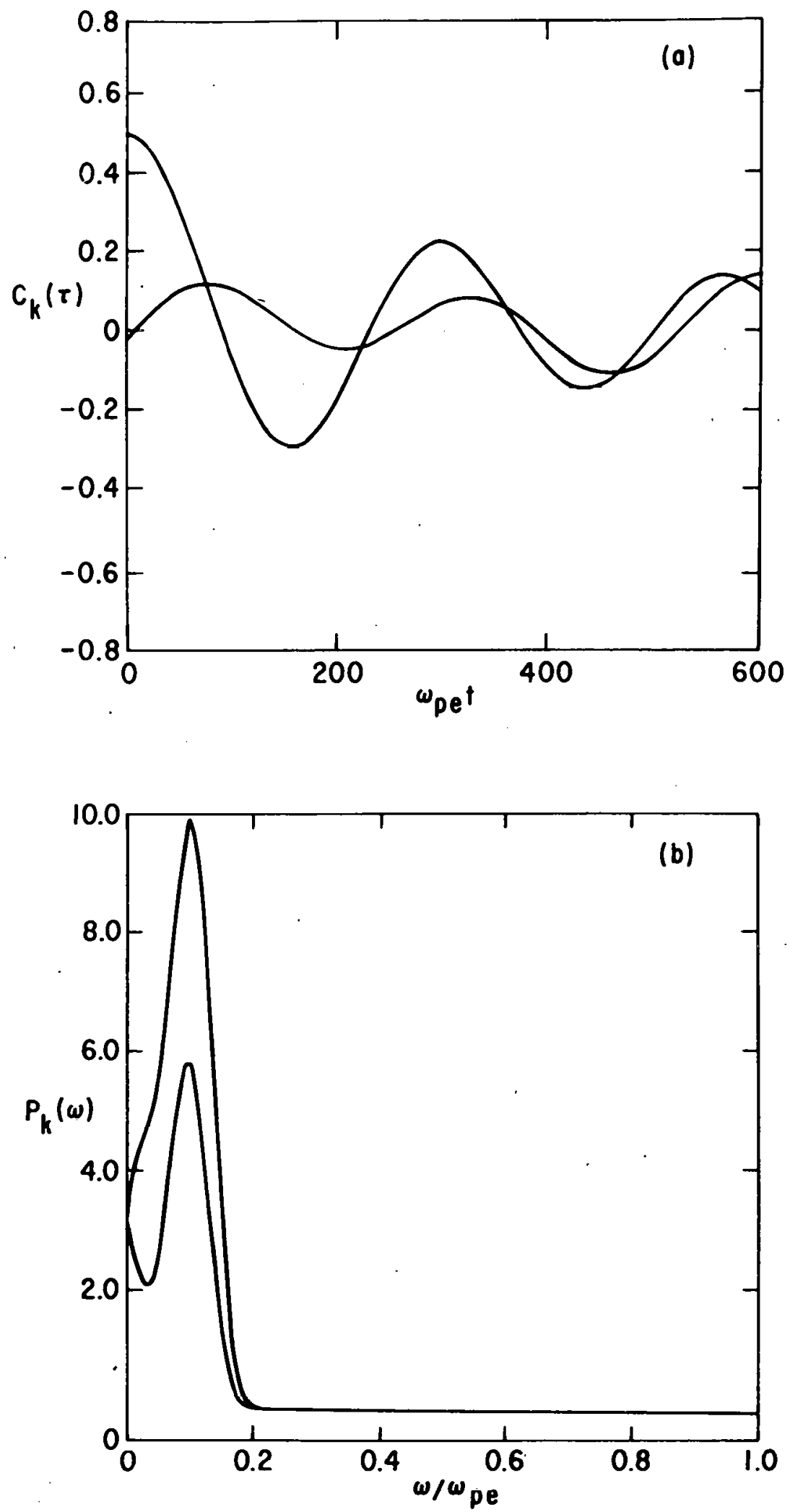

762382

Fig. 11. Correlation function of the electric field for the fourth mode (a) and its frequency spectrum (b). 

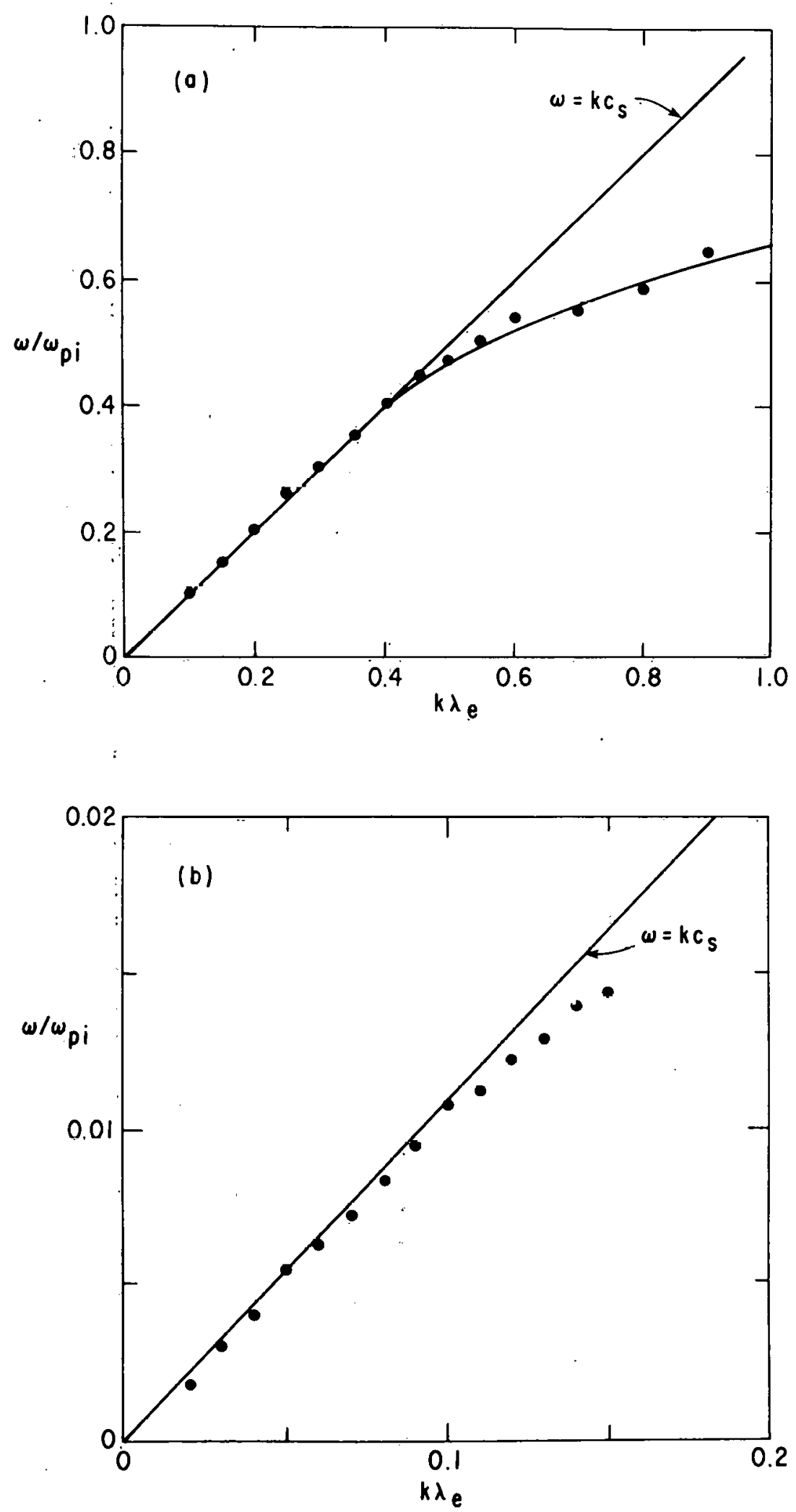

762381

Fig. 12. Dispersion relation from the measured frequency and the comparison with linear theory. 

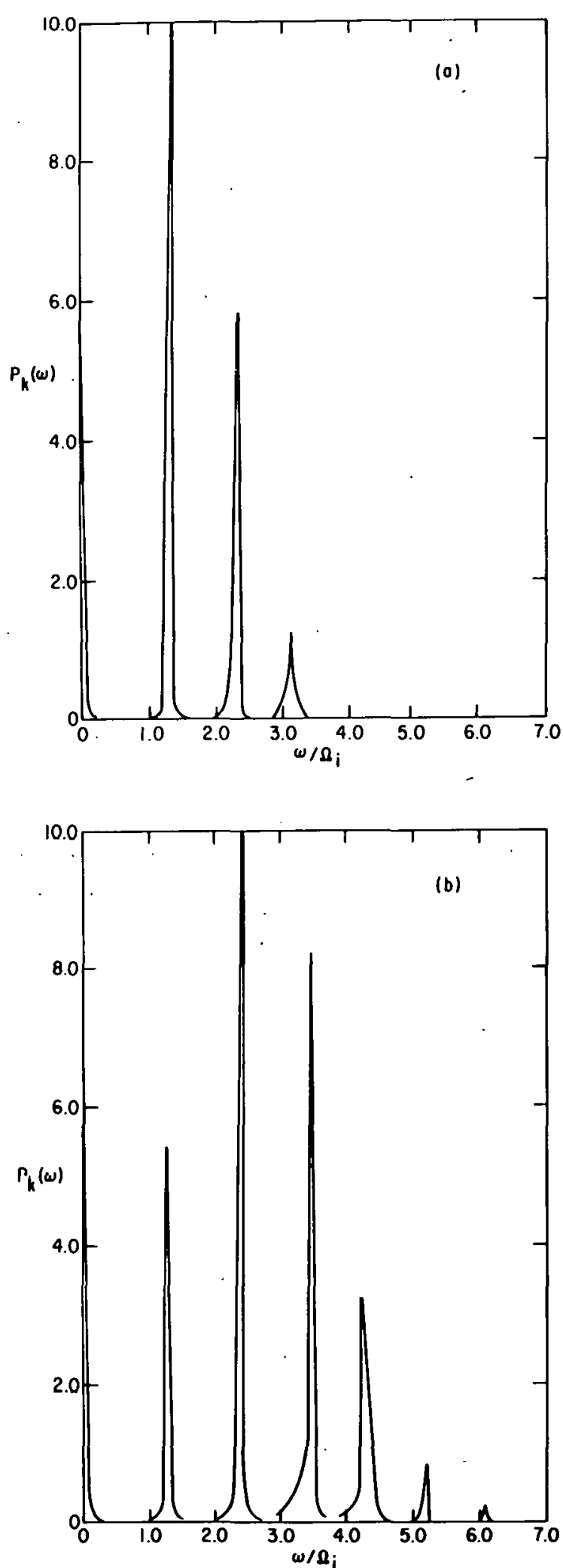

762378

Fig. 13. Frequency spectrum for the ion fluctuations in a homogeneous plasma in a magnetic field $k_{z} / k_{y}=0.02$ and $k_{y} \rho_{i}=1$ (a) and $k_{y} \rho_{i}=1.5$ (b): 

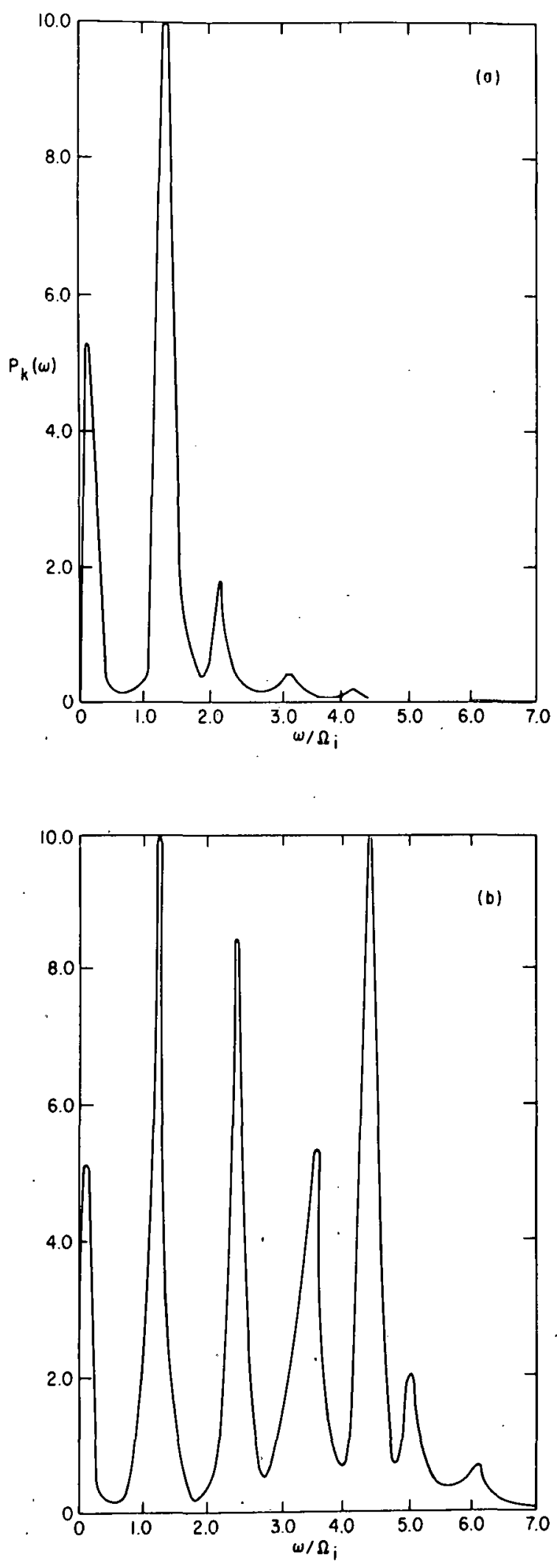

$$
6
$$

)

762377

Fig. 14. Frequency spectrum in an inhomogeneous plasma. $\mathrm{k}_{z} \mathrm{k}_{\mathrm{y}}=0.02$ and $\mathrm{k}_{\mathrm{y}} \rho_{i}=0.5$ (a) and $\mathrm{k}_{\mathrm{y}} \rho_{\mathrm{i}}=1.5$ (b). 